ARTICLE

DOI: $10.1038 / \mathrm{s} 41467-017-02191-y$

\title{
Albumin/vaccine nanocomplexes that assemble in vivo for combination cancer immunotherapy
}

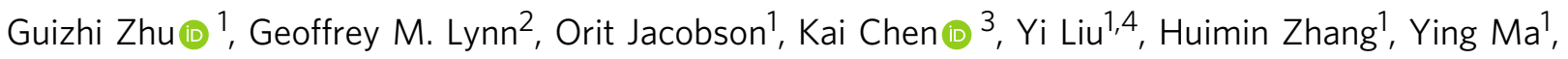
Fuwu Zhang ${ }^{1}$, Rui Tian1, Qianqian Ni ${ }^{1}$, Siyuan Cheng ${ }^{1}$, Zhantong Wang1,5, Nan Lu1, Bryant C. Yung ${ }^{1}$, Zhe Wang ${ }^{1}$, Lixin Lang ${ }^{1}$, Xiao Fu ${ }^{6}$, Albert Jin (1) ${ }^{6}$, Ido D. Weiss ${ }^{7}$, Harshad Vishwasrao ${ }^{8}$, Gang Niu', Hari Shroff ${ }^{8,9}$, Dennis M. Klinman ${ }^{10}$, Robert A. Seder ${ }^{2} \&$ Xiaoyuan Chen (1) ${ }^{1}$

Subunit vaccines have been investigated in over 1000 clinical trials of cancer immunotherapy, but have shown limited efficacy. Nanovaccines may improve efficacy but have rarely been clinically translated. By conjugating molecular vaccines with Evans blue (EB) into albuminbinding vaccines (AlbiVax), here we develop clinically promising albumin/AlbiVax nanocomplexes that self-assemble in vivo from AlbiVax and endogenous albumin for efficient vaccine delivery and potent cancer immunotherapy. PET pharmacoimaging, super-resolution microscopies, and flow cytometry reveal almost 100-fold more efficient co-delivery of CpG and antigens (Ags) to lymph nodes (LNs) by albumin/AlbiVax than benchmark incomplete Freund's adjuvant (IFA). Albumin/AlbiVax elicits $\sim 10$ times more frequent peripheral antigenspecific $C D 8^{+}$cytotoxic T lymphocytes with immune memory than IFA-emulsifying vaccines. Albumin/AlbiVax specifically inhibits progression of established primary or metastatic EG7. OVA, B16F10, and MC38 tumors; combination with anti-PD-1 and/or Abraxane further potentiates immunotherapy and eradicates most MC38 tumors. Albumin/AlbiVax nanocomplexes are thus a robust platform for combination cancer immunotherapy.

\footnotetext{
${ }^{1}$ Laboratory of Molecular Imaging and Nanomedicine, National Institute of Biomedical Imaging and Bioengineering (NIBIB), National Institutes of Health (NIH), Bethesda, MD 20892, USA. ${ }^{2}$ Vaccine Research Center, National Institute of Allergy and Infectious Diseases (NIAID), NIH, Bethesda, MD 20892, USA. ${ }^{3}$ Molecular Imaging Center, Department of Radiology, Keck School of Medicine, University of Southern California, Los Angeles, CA 90033 , USA.

${ }^{4}$ School of Engineering, China Pharmaceutical University, Nanjing 210009, China. 5 State Key Laboratory of Molecular Vaccinology and Molecular Diagnostics, Center for Molecular Imaging and Translational Medicine, School of Public Health, Xiamen University, Xiamen 361102, China. ${ }^{6}$ Laboratory of Cellular Imaging and Macromolecular Biophysics, NIBIB, NIH, Bethesda, MD 20892, USA. ${ }^{7}$ Laboratory of Molecular Immunology, NIAID, NIH, Bethesda, MD 20892, USA. ${ }^{8}$ Advanced Imaging and Microscopy Resource, National Institutes of Health, Bethesda, 20892 MD, USA. ${ }^{9}$ Section on High Resolution Optical Imaging, NIBIB, NIH, Bethesda, MD 20892, USA. ${ }^{10}$ Cancer and Inflammation Program, National Cancer Institute, Frederick, MD 21702, USA.

Correspondence and requests for materials should be addressed to X.C. (email: shawn.chen@nih.gov)
} 
T he past decade has witnessed remarkable advances in cancer immunotherapy, including immune checkpoint inhibitors ${ }^{1}$. However, only limited patient populations respond to single immune checkpoint inhibitors, and although combining multiple biologic checkpoint inhibitors increases response rates, it also elevates toxicity. Vaccines exploit synergistic signaling pathways for combination cancer immunotherapy. Despite the tremendous potential of subunit vaccines for cancer immunotherapy, their clinical outcome thus far has been suboptimal, largely due to inefficient co-delivery of adjuvants and Ags to secondary lymphoid organs, such as LNs where immune responses of lymphocytes are coordinated, leading to weak immunostimulation and immune tolerance ${ }^{2,3}$. Although tumorspecific neoantigens are promising for personalized immunotherapy ${ }^{4-9}$, there remains a lack of a general technology to deliver heterogenous peptide neoantigens efficiently. As a clinical benchmark, subunit Ags are administered in depot-forming water-in-oil emulsions (e.g., IFA) of CpG oligodeoxynucleotide, a Toll-like receptor 9 (TLR9) agonist, and T helper 1 enhancer ${ }^{10,11}$. Despite sustainable vaccine release from the depot and improved Ag immunogenicity, IFA has limited clinical efficacy. Indeed, IFA sequesters minimal Ag determinant-specific T cells in the depots,

a
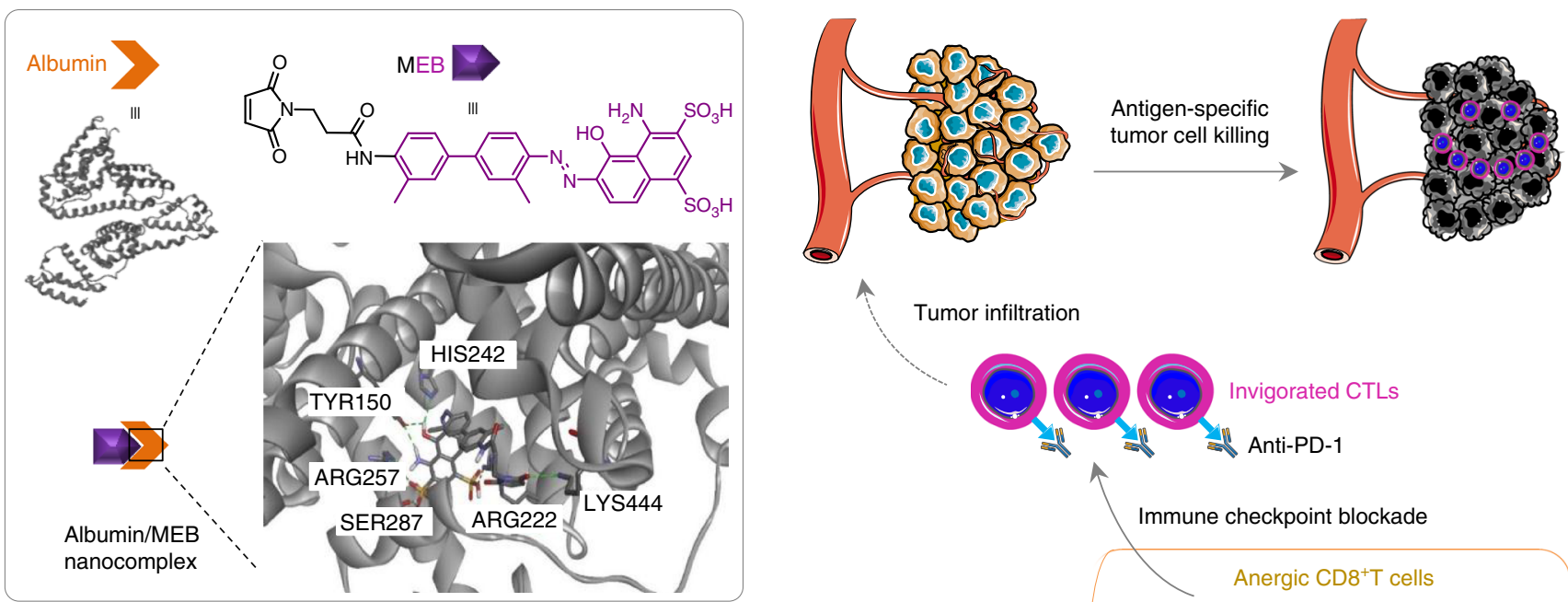

Tumor infiltration
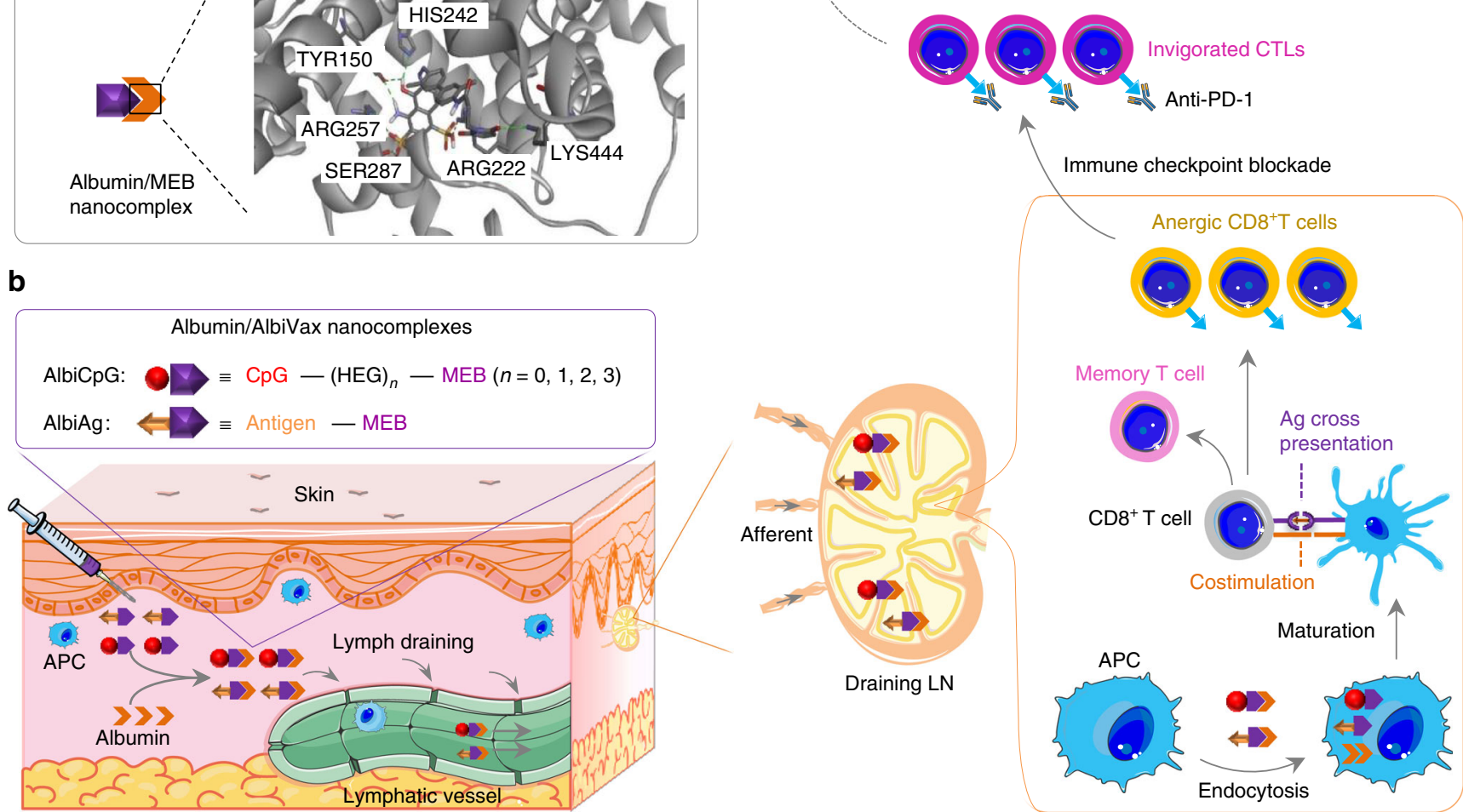

Quantitative PET pharmacoimaging in mice

Light-sheet fluorescence imaging in LNs

Super-resolution microscopy in APCs

Fig. 1 Schematic of albumin/AlbiVax nanocomplexes for efficient vaccine delivery and combination cancer immunotherapy. a Upper: structure of HSA (PDB ID: 2BXH) and chemical structure of MEB; lower: schematic structure of albumin/MEB nanocomplexes (left) and 3D molecular structure predicted by molecular docking (right). Sticks represent MEB and the amino acid residues in the binding site I of HSA. Green dashed lines represent hydrogen bonds between MEB and amino acids. $\mathbf{b}$ Working mechanism of albumin/AlbiVax nanocomplexes as potent $T$ cell vaccines. Left box: modular structures of AlbiCpG and albumin-binding Ag (AlbiAg). AlbiCpG were engineered by site specifically conjugating MEB and thiol-modified CpG, with hexaethyloxyglycol (HEG) as tunable linkers; AlbiAg was synthesized by conjugating MEB and cysteine-modified Ags, including TAA and tumor-specific neoantigen discovered via exome sequencing. Left lower: locally administered AlbiVax binds to endogenous albumin and assembles into albumin/AlbiVax nanocomplexes, which were efficiently delivered to LNs due to lymphatic drainage and prolonged retention in LNs. Right: harnessing the endocytosis pathway of albumin, albumin/AlbiCpG and albumin/AlbiAg nanocomplexes were co-delivered into APCs and activated APCs for antigen cross presentation and clonal expansion of antigen-specific CD8 ${ }^{+} \mathrm{CTLs}$, thereby eliciting robust and durable antitumor immunity. While albumin/AlbiVax nanocomplexes upregulated the expression of PD-1 on these CD8 ${ }^{+}$CTLs, combination of albumin/AlbiVax nanocomplexes with anti-PD-1 dramatically enhanced immunotherapeutic efficacy in established primary and metastatic tumors. The pharmacological behaviors of albumin/AlbiVax nanocomplexes were studied by quantitative PET imaging, light sheet fluorescence microscopy in whole cleared tissue, and super-resolution imaging in single APCs 
a

$\mathrm{CpG}\left(5^{\prime} \longrightarrow 3^{\prime}\right)$

TCCATGACGTTCCTGACGTT $-(\mathrm{HEG})_{m}-(\mathrm{PEG})_{n}-\mathrm{R}$

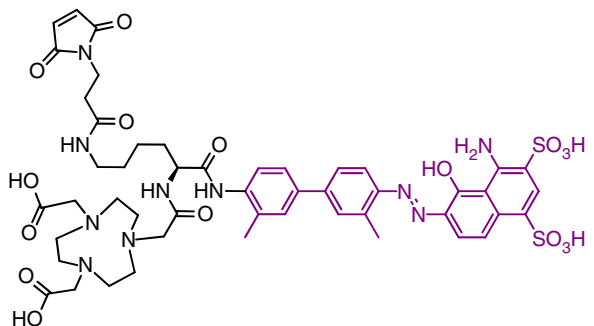

\begin{tabular}{lllll}
\hline Derivatives & $m$ & $n$ & $\mathrm{R}$ & IFA \\
\hline CpG & 0 & 0 & NOTA & No \\
IFA(CpG) & 0 & 0 & NOTA & Yes \\
PEG-CpG & 0 & 1 & NOTA & No \\
MC & 0 & 0 & NMEB & No \\
$\mathrm{MH}_{1} \mathrm{C}$ & 1 & 0 & NMEB & No \\
$\mathrm{MH}_{2} \mathrm{C}$ & 2 & 0 & NMEB & No \\
$\mathrm{MH}_{3} \mathrm{C}$ & 3 & 0 & NMEB & No \\
\hline
\end{tabular}

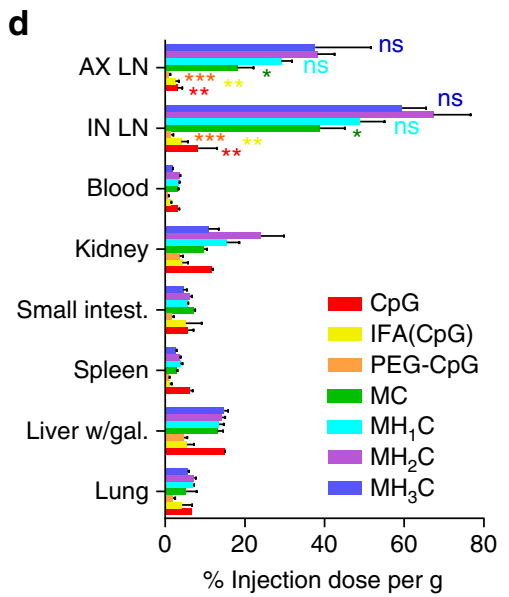

b Time post injection (h)

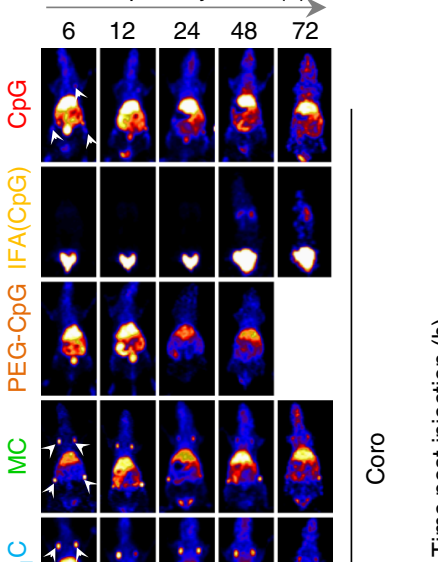

C
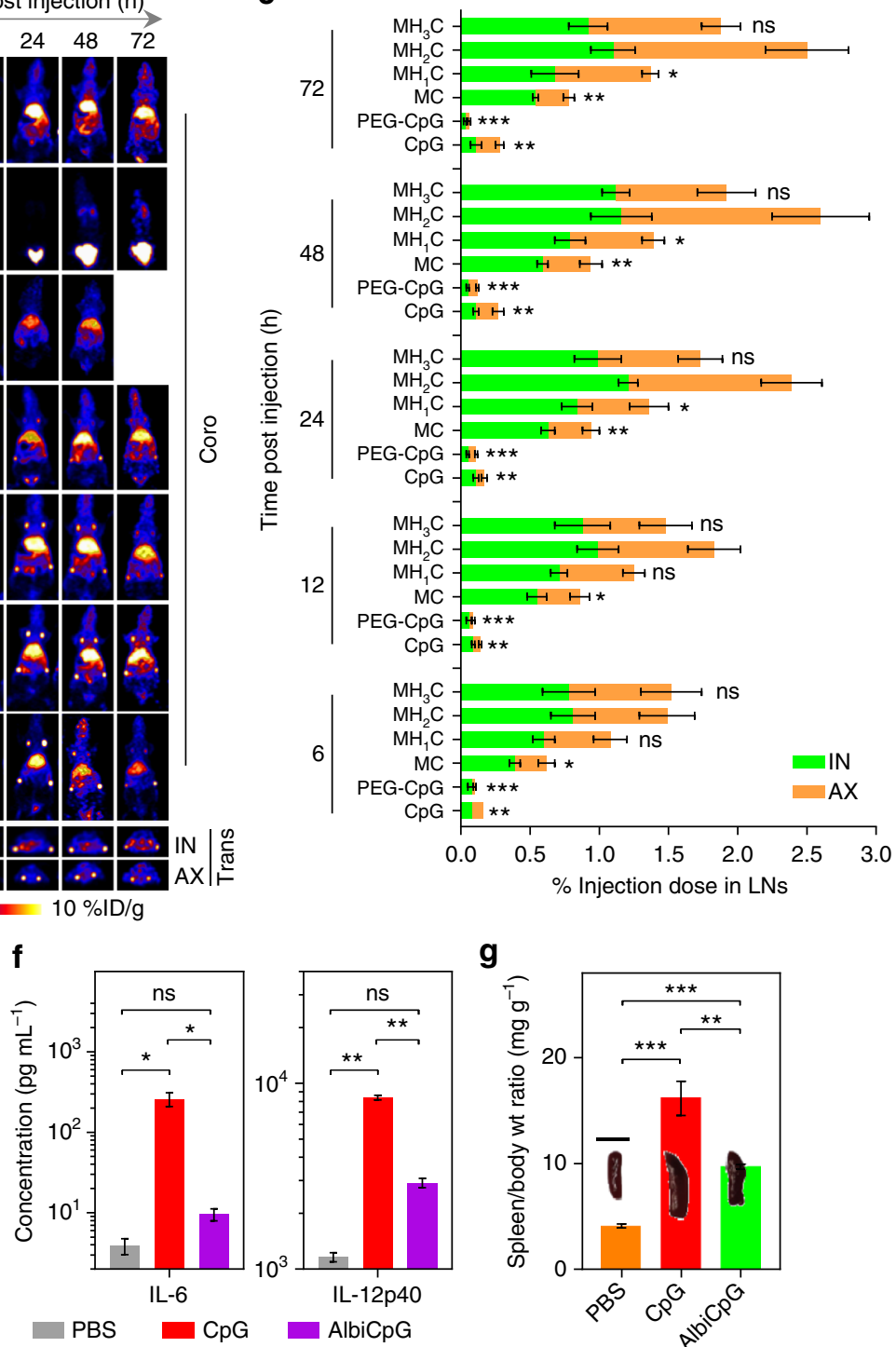

g

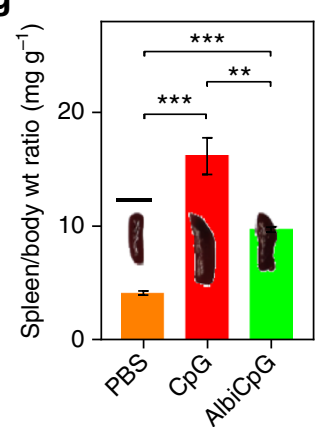

e

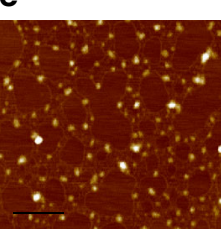

Fig. 2 Quantitative screening of albumin/AlbiCpG nanocomplexes for LN delivery. a Structures, radiolabeling, and formulations of CpG derivatives for PETbased screening. Shown in the middle is the molecular structure of NMEB used for radiolabeling of AlbiCpG. Bioconjugations were conducted using thiol on $\mathrm{Cp}$, maleimide on NMEB (for AlbiCpG), or NOTA (for $\mathrm{CpG}$ and IFA(CpG)), as well as maleimide and alkyne on bifunctional PEG20K and azide on NOTA for PEG-CpG. b Upper: representative coronal (coro) PET images showing FVB mice at $6 \mathrm{~h}$ post s.c. injection (dose: 4.4-5.5 Mbq) of CpG derivatives at tail base. Nanocomplexes of albumin with four MEB-CpG derivatives, respectively, efficiently delivered CpG to LNs, relative to free CpG, PEG-CpG, and IFA $(\mathrm{CpG})$. Lower: representative transverse (trans) PET images showing LN delivery of $\mathrm{MH}_{2} \mathrm{C}$ at $6 \mathrm{~h}$ post injection. White arrow heads mark IN and AX LNs. c Amounts of $\mathrm{CpG}$ derivatives in IN and AX LNs quantified from three-dimensional (3D)-reconstructed, decay-corrected PET images $(n=4-8)$. The IFA $(\mathrm{CpG})$ signal in LNs was too low to visualize for quantification. $\mathbf{d}$ Biodistribution of tested compounds in resected organs measured by $\gamma$ counting 3 days post injection $(n=4)$. (Liver/gal: liver with gallbladder; intest.: intestine). e An AFM image of HSA/AlbiCpG nanocomplexes (premixed AlbiCpG: HSA = 1:1). Scale bar: $200 \mathrm{~nm} . \mathbf{f}, \mathbf{g}$ In C57BL/ 6 mice, AlbiCpG s.c. injected at the tail base ameliorated the systemic toxicity of CpG $(n=4)$ as shown by lower IL-6 and IL-12p40 titers in blood (f) (dose: $5 \mathrm{nmol} \mathrm{CpG} \mathrm{equivalents)} \mathrm{and} \mathrm{ameliorated} \mathrm{splenomegaly} \mathrm{(g)} \mathrm{on} \mathrm{day} 6$ (dose: $5 \mathrm{nmol} C \mathrm{CpG}$ equivalents on day 0 and day 3). Scale bar for spleen: $1 \mathrm{~cm}$. Wt: weight. Data show mean \pm s.e.m. of two-three independent experiments. ${ }^{\star \star \star} p<0.001,{ }^{\star \star} p<0.01$, ${ }^{\star} p<0.05$, ns: not significant $(p>0.05)$ by one-way ANOVA with Bonferroni post test. Asterisks in $\mathbf{c}$ indicate statistically significant differences between the corresponding compounds with $\mathrm{MH}_{2} \mathrm{C}$

exhausting and depleting $\mathrm{T}$ cells, thereby preventing $\mathrm{T}$ cells from infiltrating tumors ${ }^{12}$. Alternatively, nanovaccines, which can efficiently co-deliver locally administered adjuvants/Ags into LNs via lymphatics, have been explored for vaccine delivery ${ }^{13-16}$. However, the pharmacological behaviors of perhaps all currently reported nanovaccines were studied either invasively or semiquantitatively, and more importantly, the clinical translation of most synthetic nanovaccines has been hampered by complications in large-scale manufacturing, quality control, formulation, and safety ${ }^{17,18}$.

We hypothesize that nanovaccines assembled in vivo from exogenous molecular vaccines and endogenous nanocarriers would not only enhance vaccine bioavailability in LNs, but also bypass the complications mentioned above. Compared with synthetic nanovaccines, molecular vaccines are chemically defined and often relatively well suited to large-scale production, 
including quality control and safety evaluation. Many natural biomolecules, such as albumin and immunoglobins (IgGs), are excellent candidates for endogenous carriers, given their (1) long half-lives (over 20 days in humans) ${ }^{19}$, (2) ubiquity in peripheral tissues, and (3) easy accessibility to vaccines with good patient compliance $^{20}$. Internalized $\operatorname{IgG}$ and albumin in cells can be recycled from endolysosomes and transported out of cells via acidic binding to receptors, including fragment crystallizable receptor $(\mathrm{FcR})$ and neonatal $\mathrm{Fc}$ receptor $(\mathrm{FcRn})$, thereby avoiding protein degradation and prolonging their half-lives. We chose albumin as an endogenous component to assemble nanovaccines with exogenous molecular vaccines, for the following reasons: (1) While binding to endogenous IgG Fc likely interferes with IgG's biological functions, albumin is a natural carrier with multiple, versatile, intrinsic-binding sites for biomolecules, and drugs ${ }^{21-25}$; (2) The size of murine and human albumins $(66 \mathrm{kDa})$ exceeds the cutoff $(45 \mathrm{kDa})$ to be disseminated systemically from interstitial space by blood ${ }^{26}$, meaning that nearly all will be drained through lymphatics to LNs. Together with the slow lymph flow, these characteristics offer a long time window for albumin/AlbiVax nanocomplexes to modulate lymphocytes in LNs. Interestingly, albumin can be efficiently internalized by antigen-presenting cells (APCs) via endocytosis, which can facilitate intracellular vaccine delivery for optimal Ag processing and presentation. Indeed, albumin-drug conjugates/complexes have been enthusiastically pursued for decades ${ }^{27}$, ranging from albumin conjugates of antiviral 5-fluorodeoxyuridine and cytosine arabinoside $22,25,28$, lipid-prodrug conjugates ${ }^{29}$, drug-peptide/fatty acid conjugates ${ }^{30}$, to Abraxane of albumin/paclitaxel nanocomplexes. Specific albumin-vaccine conjugates include streptococcal protein Gantigen conjugates ${ }^{31}$, albumin-antigen/interleukin-2 (IL-2) fusion proteins ${ }^{25,28}$, and lipid-vaccine conjugates ${ }^{20,32}$ that remarkably improved vaccine delivery to LNs and substantially potentiated immune responses.

Here we took a clinically oriented perspective to develop AlbiVax by first repurposing a clinically practiced EB that binds to albumin at binding site $\mathrm{I}^{33}$, and then conjugating molecular vaccines with an $\mathrm{EB}$ derivative. The resulting albumin/AlbiVax nanocomplexes that assemble in vivo were efficiently delivered to LNs and induced potent and durable anti-cancer immunity. EB and derivatives have been developed for preclinical and clinical LN identification ${ }^{34-39}$. They have excellent clinical safety profiles ${ }^{36,39}$, and rhesus monkeys survived $25 \mathrm{mg} \mathrm{kg}^{-1}$ systemically injected $\mathrm{EB}^{40}$, a dose 500 -fold higher than that of AlbiVax. We exploited multiscale pharmacoimaging to quantitatively and systematically investigate the pharmacology of albumin/AlbiVax nanocomplexes in animals by PET, in entire LNs by light sheet fluorescence microscopy, and in APCs by super-resolution instantaneous structured illumination microscopy (instant SIM) 41 . Albumin/AlbiVax nanocomplexes potentiated both innate and adaptive immunity, leading to a markedly expanded repertoire of Ag-specific $\mathrm{CD}^{+}$CTLs and $\mathrm{T}$ cell memory. Albumin/AlbiVax nanocomplexes, alone or in combination with anti-PD-1 and/or Abraxane, dramatically inhibited tumor progression in multiple syngeneic tumor models.

\section{Results}

Efficient LN delivery of albumin/AlbiVax nanocomplexes. AlbiVax was synthesized by conjugating thiol-modified vaccines with maleimide-functionalized EB derivative (MEB), which tightly binds to human serum albumin (HSA) on binding site I (Fig. 1 and Supplementary Figs. 1 and 2). CpG was studied first. MEB was conjugated onto the 3 '-end of CpG (Supplementary Fig. 3), and the resulting MEB-CpG, as a candidate of albuminbinding CpG (AlbiCpG), either alone or premixed with albumin, maintained the immunostimulatory activity of CpG in RAW264.7 macrophages. By contrast, MEB modification on the $5^{\prime}$-end of CpG abrogated its activity ${ }^{42}$. MEB-GpC control was also not immunostimulatory (Supplementary Fig. 4). Like $\mathrm{MEB}^{43}$, in the presence of albumin, the originally self-quenched MEB fluorescence of MEB-CpG was recovered and the fluorescence lifetime was prolonged, indicating albumin binding of MEB-CpG. The fluorescence of MEB-CpG was less than MEB upon albumin binding (Supplementary Fig. 5a, b). Intriguingly, the fluorescence of MEB-DNAs was partially activated in the absence of albumin, in a manner independent of DNA sequence, length, and deoxynucleotides, presumably resulting from intramolecular electrostatic interactions between MEB and ssDNA in MEB-DNAs, as well as reduced intermolecular stacking of MEB-DNAs relative to free MEB (Supplementary Figs. 6-9).

The above fluorescence phenomena imply interactions between MEB and DNA in MEB-CpG, which likely interfere with the albumin binding of MEB-CpG. Thus, 0,1 , 2, and 3 units of HEG linkers between $\mathrm{MEB}$ and $\mathrm{CpG}$ were used to synthesize MEB-CpG (MC), MEB-HEG-CpG $\left(\mathrm{MH}_{1} \mathrm{C}\right)$, MEB$(\mathrm{HEG})_{2}-\mathrm{CpG}\left(\mathrm{MH}_{2} \mathrm{C}\right)$, and $\mathrm{MEB}-(\mathrm{HEG})_{3}-\mathrm{CpG}\left(\mathrm{MH}_{3} \mathrm{C}\right)$ with ca. $0,2,4$, and 6-nm linkers, respectively, and quantitatively screened for LN delivery by PET in FVB mice. Specifically, MEB-CpG derivatives were radiolabeled with ${ }^{64} \mathrm{Cu}\left(t_{1 / 2}: 12.6 \mathrm{~h}\right)$ via 1,4,7-triazacyclononane-triacetic acid (NOTA)-MEB $(\mathrm{NMEB})^{44}$ (Fig. 2a and Supplementary Fig. 10), and were subcutaneously (s.c.) injected (dose: $4.4-5.5 \mathrm{Mbq}$ ) at the tail base of mice, followed by PET imaging over 3 days (Fig. 2b, Supplementary Figs. 11 and 12, and Supplementary Movie 1). PEG-CpG conjugate (PEG MW: $20 \mathrm{kDa}$ ), and CpG with or without IFA were used as controls. As quantified from decaycorrected PET results (Fig. 2c), only $<0.3 \%$ injection dose (\%ID) of CpG was delivered to inguinal (IN) and axillary (AX) LNs, and IFA-emulsified CpG [IFA(CpG)] was substantially trapped at the injection sites with little delivered to draining LNs. In contrast, all MEB-CpG derivatives were efficiently delivered to LNs (IN + $\mathrm{AX}$ ), with $\mathrm{MH}_{2} \mathrm{C}$ and $\mathrm{MH}_{3} \mathrm{C}$ being the most efficient (\%ID: 0.62 MC, $1.08 \mathrm{MH}_{1} \mathrm{C}, 1.49 \mathrm{MH}_{2} \mathrm{C}$, and $1.52 \mathrm{MH}_{3} \mathrm{C}$ at $6 \mathrm{~h}$ post injection). $\mathrm{MH}_{2} \mathrm{C}$ peaked with $2.60 \pm 0.30 \% \mathrm{ID}$ at $48 \mathrm{~h}$ post injection, with more than 1.45 \% ID retained in LNs over 3 days. The area under the curve (AUC) of $\mathrm{MC}, \mathrm{MH}_{1} \mathrm{C}, \mathrm{MH}_{2} \mathrm{C}$, and $\mathrm{MH}_{3} \mathrm{C}$ in these LNs were 3.0-, 5.5-, 6.5-, and 6.1-fold greater, respectively, than that of $\mathrm{CpG}$ within 3 days. The deep penetration of PET allowed to observe substantial amounts of MEB-CpG derivatives also in deep iliac (IL) LNs (Supplementary Fig. 13). PEG-CpG failed to enhance LN delivery, which suggests the essential and unique role of albumin for efficient LN delivery of albumin/AlbiVax nanocomplexes and rules out that the large size of albumin/AlbiCpG nanocomplexes was the sole factor contributing to efficient LN delivery. Significant amount of CpG and derivatives were still retained at injection sites (Supplementary Fig. 14), likely due to the slow lymphatic clearance and the interaction of phosphorothioate DNA with extracellular matrix. Consistently, high signal intensity and density of MEB-CpG derivatives in resected draining LNs were detected by $\gamma$ counting. On day 3, IN LNs had $38.8 \pm 6.3,48.9 \pm 6.2,67.4 \pm 9.3$, and 59.5 \pm 6.02 (mean \pm standard error of mean (s.e.m.), same below; $n=$ 4) percent injection dose per gram of tissue $(\% \mathrm{ID} / \mathrm{g})$ of $\mathrm{MC}$, $\mathrm{MH}_{1} \mathrm{C}, \mathrm{MH}_{2} \mathrm{C}$, and $\mathrm{MH}_{3} \mathrm{C}$, respectively, in contrast to $8.3 \pm 4.7$, $4.0 \pm 1.7$, and $1.48 \pm 0.55$ (mean \pm s.e.m.; $n=4) \% \mathrm{ID} / \mathrm{g}$ of $\mathrm{CpG}$, IFA(CpG), and PEG-CpG, respectively (Fig. 2d, Supplementary Fig. $15 \mathrm{a}-\mathrm{c})$. On day5, $2.5 \pm 1.3,37.3 \pm 6.1,38.6 \pm 3.4,37.6 \pm 1.8$ $(n=4) \% \mathrm{ID} / \mathrm{g}$ of $\mathrm{CpG}, \mathrm{MH}_{1} \mathrm{C}, \mathrm{MH}_{2} \mathrm{C}$, and $\mathrm{MH}_{3} \mathrm{C}$, respectively, were detected in IN LNs (Supplementary Fig. 15d, e). MEB-CpG derivatives were also observed in organs such as liver, kidney, and small intestine, presumably because of the ubiquity of albumin as 
a

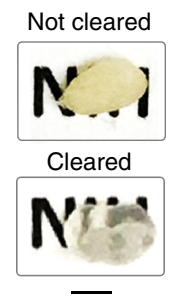

b

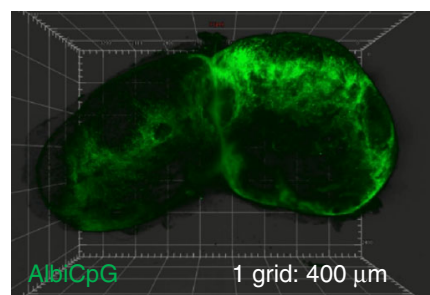

C

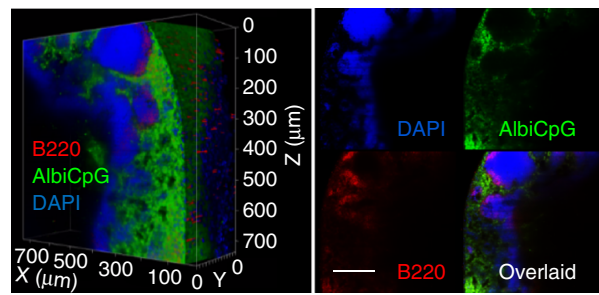

d

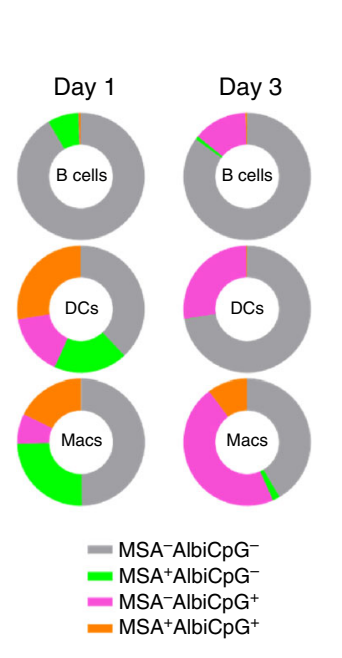

e

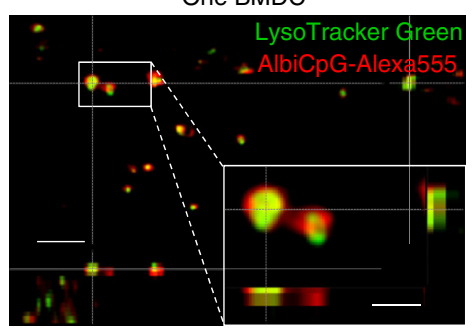

One BMDC f

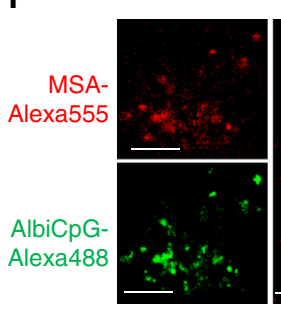

g

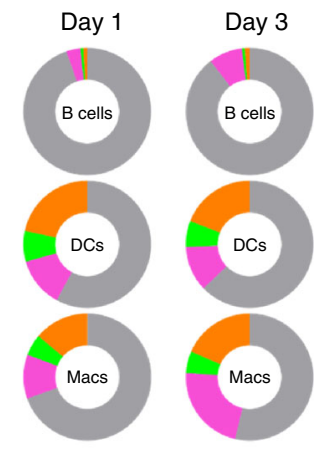

- AlbiCpG-AlbiSIINFEKL- AlbiCpG ${ }^{+}$AlbiSIINFEKL- AlbiCpG-AlbiSIINFEKL ${ }^{+}$ AlbiCpG ${ }^{+}$AlbiSIINFEKL ${ }^{+}$ h

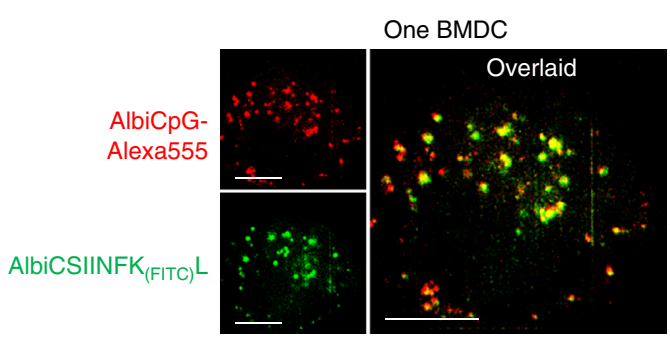

i

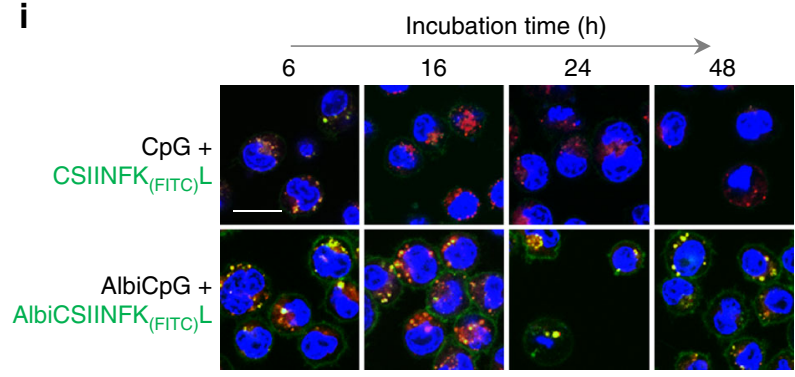

Fig. 3 Super-resolution observation of intranodal and intracellular co-delivery of albumin/AlbiVax nanocomplexes. a Photographs of non-cleared and PACT-cleared mouse LNs. Scale bar: $2 \mathrm{~cm}$. b, c Light sheet fluorescence microscopy images showing the 3D intranodal distribution of albumin/ AlbiCpG-Alexa488 nanocomplexes in a whole LN (b) and a close-up of the subcapsular sinus areas (c left: 3D distribution; right: cross sections) 1 day after injection. Substantial albumin/AlbiCpG nanocomplexes were located within or near the subcapsular sinus and around B cell follicles (Supplementary Videos 2 and 3). Scale bar in b: $400 \mu \mathrm{m}$; Scale bar in c: $200 \mu \mathrm{m}$. d Fractions of IN LN B220 ${ }^{+}$B cells, CD11c ${ }^{+}$DCs, and F4/80 ${ }^{+}$macrophages that took up AlbiCpG-Alexa555 and/or MSA-FITC, on day 1 and day 3 post s.c. injection of premixed AlbiCpG + MSA into C57BL/6 mice $(n=4)$. e Deconvoluted confocal microscopy images showing AlbiCpG $(200 \mathrm{nM})$ in the endolysosomes of one single BMDC after 2-h incubation. Inset: 2 endolysosomes showing AlbiCpG primarily on the endolysosome membrane. (Red: AlbiCpG; green: LysoTracker Green.) Scale bar: $2 \mu \mathrm{m}$; inset: $500 \mathrm{~nm}$. f Instant SIM superresolution images showing co-localization of MSA-Alexa555 (3 $\left.\mathrm{mg} \mathrm{mL}^{-1}\right)$ and AlbiCpG-Alexa488 (200 nM) in one BMDC after 2-h incubation. Scale bars: $4 \mu \mathrm{m}$. $\mathbf{g}$ Fractions of IN LN B220 ${ }^{+}$B cells, CD11c ${ }^{+}$DCs, and F4/80+ macrophages that took up AlbiCpG-Alexa555 and AlbiCSIINFEK(FITC) on day 1 and day 3 post s.c. injection of AlbiCpG + AlbiSIINFEKL into C57BL/6 mice $(n=4)$. $\mathbf{h}$ Instant SIM images showing intracellular co-delivery of AlbiCpG-Alexa555 $(200 \mathrm{nM})+$ AlbiCSIINFEK $_{(\mathrm{FITC})} \mathrm{L}(200 \mathrm{nM})$ in one BMDC after 2-h incubation. AlbiCSIINFEK ${ }_{(\mathrm{FITC})} \mathrm{L}$ was located together with AlbiCpG as well as separately in the cytosol. Scale bars: $2 \mu \mathrm{m}$. i Confocal microscopy images showing efficient antigen presentation of AlbiCSIINFEK (FITC) $\mathrm{L}(500 \mathrm{nM})+\mathrm{AlbiCpG}(500$ $\mathrm{nM})$, compared with CSIINFEK $(\mathrm{FITC}) \mathrm{L}(500 \mathrm{nM})+\mathrm{CpG}(500 \mathrm{nM})$ in BMDCs. For incubation time longer than $6 \mathrm{~h}$, fresh medium was substituted after 8-h incubation. (Blue: Hoechst 33342; green: FITC; red: LysoTracker Red.) Scale bars: $10 \mu \mathrm{m}$

well as the metabolism and renal clearance of $\mathrm{CpG}$ derivatives. Taken together, $\mathrm{MH}_{2} \mathrm{C}$, which showed strong binding affinity with mouse serum albumin (MSA) $\left(K_{\mathrm{d}}=1.0 \mu \mathrm{M} ; R^{2}=0.90\right)$ (Supplementary Fig. 16), was selected as the optimized AlbiCpG for further studies (Supplementary Fig. 11g). The efficient delivery of AlbiCpG was also verified by the purplish MEB color and strong MEB fluorescence of draining LNs (Supplementary Fig. 17). Overall, the efficient LN delivery of albumin/AlbiCpG nanocomplexes was attributed to a combination of relatively large sizes ( $d$ : $\sim 13.0 \mathrm{~nm}$; Fig. 2e and Supplementary Fig. 18), prolonged LN retention and efficient uptake by APCs (discussed below). Worth noting, AlbiCpG elicited less serum IL-6 and IL-12p40 than free $\mathrm{CpG}$ early after injection (Fig. 2f and Supplementary Fig. 19a) and milder splenomegaly (Fig. $2 \mathrm{~g}$ and Supplementary Fig. 19b), suggesting the amelioration of acute systemic toxicity of CPG by reducing systemic dissemination ${ }^{45}$. 
a
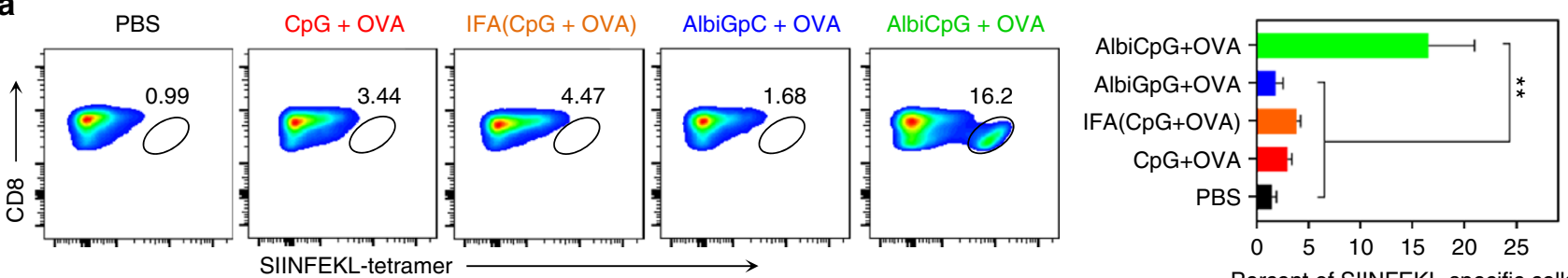

Percent of SIINFEKL-specific cells among live $\mathrm{CD}^{+} \mathrm{T}$ cells in PBMCs
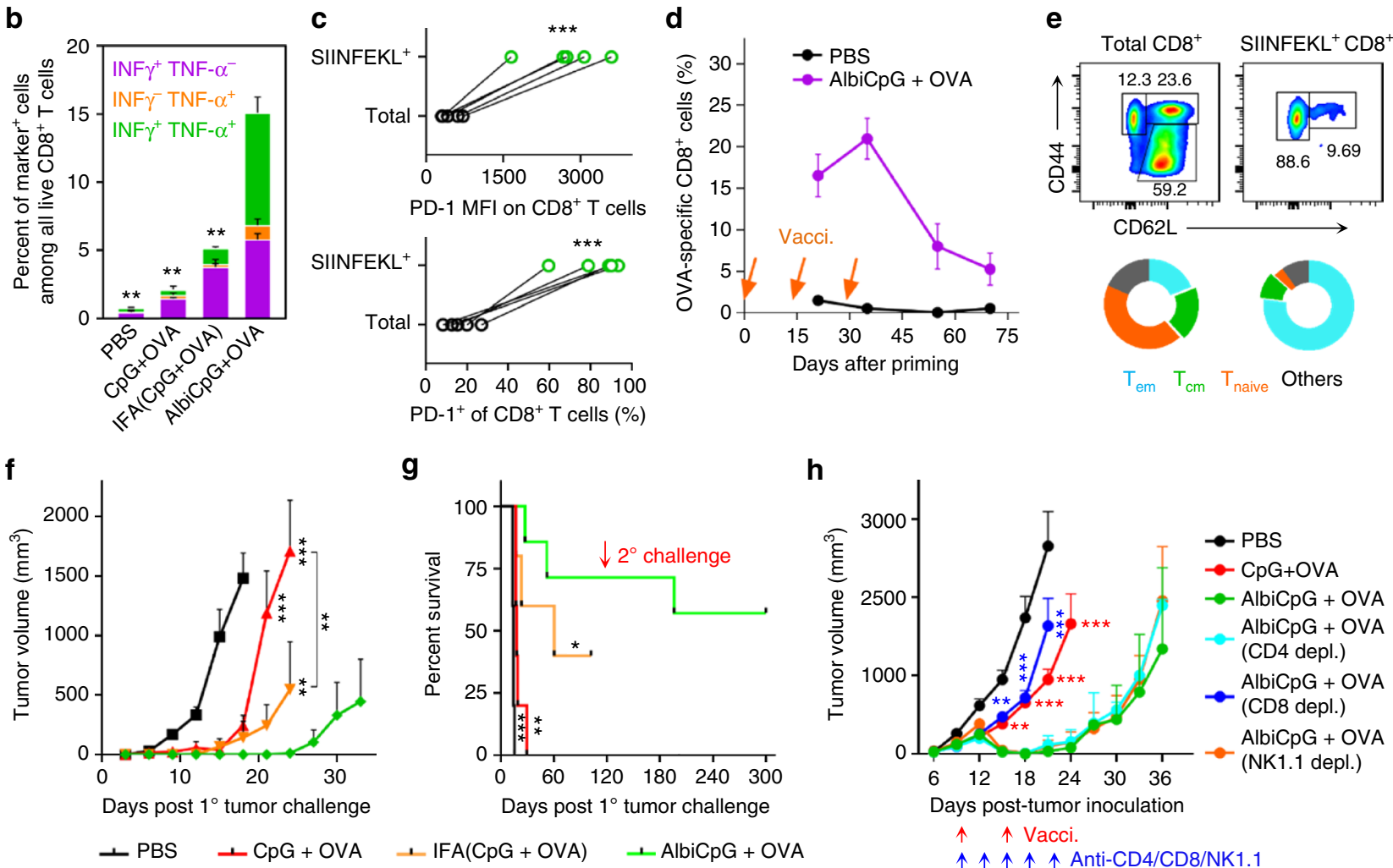

Fig. 4 Albumin/AlbiVax nanocomplexes induced potent and durable antitumor T cell responses. a-g C57BL/6 mice $(n=5-7)$ were s.c. vaccinated with AlbiVax ( $2 \mathrm{nmol}$ AlbiCpG equivalents $+10 \mu \mathrm{g}$ OVA) at the tail base on day 0, day 14, and day 28, followed by immune analysis on day 21 , day 35 , and day 70 , and $1^{\circ}$ tumor challenge on day 71. a Representative flow cytometry plots (left) and frequency (right) of SIINFEKL ${ }^{+} \mathrm{CD} 8^{+} \mathrm{T}$ cells in peripheral blood on day 21 stained using phycoerythrin (PE)-labeled $\mathrm{H}-2 \mathrm{~K}^{\mathrm{b}}$-SIINFEKL tetramer. $\mathbf{b}$ Percentage of cytokine-producing $\mathrm{CD}^{+} \mathrm{T}$ cells in peripheral blood, measured by intracellular staining of IFN- $\gamma$ and TNF- $\alpha$ on day 21. $\mathbf{c}$ Higher level (MFI) and frequency of PD-1 expression on SIINFEKL ${ }^{+} \mathrm{CD} 8 \mathrm{~T}$ cells than that on total $\mathrm{CD}^{+} \mathrm{T}$ cells in peripheral blood on day 21. (Two-tailed paired $t$ test.) $\mathbf{d}$ Frequencies of SIINFEKL ${ }^{+} \mathrm{CD} 8^{+} \mathrm{CTLs}$ in peripheral blood over 70 days post priming e Representative flow cytometry results (upper) and percentage (lower) of effector memory $T$ cells (Tem, CD62 $\mathrm{L}^{-} \mathrm{CD} 44^{+}$), central memory $T$ cells $(T c m$, $\left.\mathrm{CD} 62 \mathrm{~L}^{\text {high }} \mathrm{CD}_{4} 4^{+}\right)$, and naive T cells $\left(\mathrm{CD} 62 \mathrm{~L}^{+} \mathrm{CD} 44^{-}\right)$in peripheral blood on day 70, showing AlbiVax-induced T cell memory. $\mathbf{f}, \mathbf{g}$ Tumor growth curve $(\mathbf{f})$ and mouse survival ( $\mathbf{g}$ ) after s.c. challenging vaccinated mice with EG7.OVA cells. $1^{\circ}$ challenge: $3 \times 10^{5}$ cells on the right shoulder on day 71 post priming vaccination; $2^{\circ}$ challenge: $1 \times 10^{6}$ cells on the right flank on day 211. h AlbiCpG + OVA regressed established EG7.OVA tumor. C57BL/6 mice $(n=6-8)$ were s.c. inoculated with $3 \times 10^{5}$ EG7.OVA cells on day 0 , and treated with AlbiCpG + OVA ( $2 \mathrm{nmol}$ CpG equivalents, $20 \mu \mathrm{g}$ OVA) on day 6, day 12 , and day 18. Lymphocyte depletion by anti-CD8, but not anti-CD4 or anti-NK1.1 (200 $\mu$ g, on day 6, day 9, day 12, day 15, and day 18) abrogated the therapeutic efficacy of AlbiVax. ${ }^{\star \star \star} p<0.001,{ }^{\star \star} p<0.01,{ }^{\star} p<0.05$, ns: not significant $(p>0.05)$, by one-way ANOVA with Bonferroni post test, unless denoted otherwise. Data show mean \pm s.e.m. of two-three independent experiments. Asterisks in $\mathbf{a}, \mathbf{b}, \mathbf{f}-\mathbf{h}$ indicate statistically significant differences between AlbiCpG and other groups

Super-resolution analysis of nanovaccines in LNs and APCs. The intranodal distribution of albumin/AlbiCpG nanocomplexes was further mapped by light sheet fluorescence microscopy (LSFM), which allows for higher resolution, 3D imaging of whole tissues with low photobleaching. Specifically, 1 day post s.c. injection of AlbiCpG-Alexa488 (higher photostability of Alexa488 than MEB) in C57BL/6 mice, draining IN LNs were resected and "cleared" to be transparent using passive CLARITY technique $(\mathrm{PACT})^{46}$ (Fig. 3a). LSFM of the cleared LNs mapped the 3D distribution of intranodal AlbiCpG, which was especially abundant within or near the subcapsular sinus areas and around B cell follicles, as verified in LN slices (Fig. 3b, c, Supplementary Fig. 20, and Supplementary Movies 2 and 3).

We further analyzed the intracellular delivery of albumin/ AlbiCpG nanocomplexes in APCs, a pivotal process for potent immunomodulation. Premixed MSA-FITC and AlbiCpGAlexa555 were injected s.c. at the tail base of C57BL/6 mice. $\mathrm{B} 220^{+} \mathrm{B}$ cells, CD $11 \mathrm{c}^{+} \mathrm{DCs}$, and $\mathrm{F} 4 / 80^{+}$macrophages were analyzed by flow cytometry on day 1 and day 3 post injection (Fig. 3d and Supplementary Fig. 21a, b). On day 1, 43\% DCs and 
$27 \%$ macrophages were $\mathrm{AlbiCpG}^{+}$, and $28 \%$ DCs and $18 \%$ macrophages were AlbiCpG ${ }^{+} \mathrm{MSA}^{+}$, suggesting efficient uptake of albumin/AlbiCpG nanocomplexes. By contrast, only $<1 \%$ B cells were $\mathrm{AlbiCpG}^{+}$, despite $7 \% \mathrm{MSA}^{+} \mathrm{B}$ cells. On day 3, 27\% DCs, $55 \%$ macrophages, and $14 \% \mathrm{~B}$ cells were $\mathrm{AlbiCpG}^{+}$. MSA-FITC was only marginally detected, likely due to exhaustion. Efficient uptake of AlbiCpG was recapitulated in RAW264.7 macrophages and bone-marrow-derived dendritic cells (BMDCs) by $\gamma$ counting of ${ }^{64} \mathrm{Cu}$-labeled AlbiCpG, confocal microscopy, and flow cytometry (Supplementary Fig. 21c-e). Deconvoluted confocal microscopy revealed ubiquitous AlbiCpG-Alexa555 in BMDC endolysosomes, and intriguingly, primarily on the endolysosome membranes wherein TLR9 resides $^{47}$ (Fig. 3e, Supplementary Fig. 21f, and Supplementary Movie 4).

To study the intracellular vaccine delivery in APCs, we exploited instant SIM, a super-resolution imaging system that features ultrafast acquisition (up to $100 \mathrm{~Hz}$ ) of 3D superresolution images ${ }^{41}$. Dye-labeled MSA was rapidly endocytosed into BMDC endolysosome (Supplementary Fig. 21g), where MSA was co-localized with AlbiCpG when premixed MSA-Alexa555/ AlbiCpG-Alexa488 was added to BMDCs (Fig. 3f), recapitulating intracellular co-delivery of albumin/AlbiCpG nanocomplexes by endocytosis. Albumin/AlbiCpG nanocomplexes upregulated the expression of costimulatory factor CD80 on DCs in draining LNs of C57BL/6 mice, despite accompanying lymphadenopathy (Supplementary Fig. 22a, b). In RAW264.7 macrophages and BMDCs, albumin/AlbiCpG nanocomplexes upregulated the expression of CD80 and CD86, and potently stimulated the production of TNF $\alpha$, IL-6, and IL-12p40 (Supplementary Fig. $22 c, d)$.

We then studied co-delivery of $\mathrm{CpG}$ and Ag by albumin/ AlbiVax. We modified SIINFEKL, an epitope of ovalbumin (OVA), with cysteine for MEB conjugation and with FITC on lysine, which retained epitope binding ability to $\mathrm{H}-2 \mathrm{~K}^{\mathrm{b}} \mathrm{MHC}$ class $\mathrm{I}^{48}$. The resulting MEB-CSIINFEK (FITC) $_{\text {(denoted as }}$ AlbiCSIINFEK $_{(\mathrm{FITC})} \mathrm{L}$ ) was mixed with AlbiCpG-Alexa555 and s.c. co-injected at the tail base of C57BL/6 mice. After 1 and 3 days, IN LN DCs and macrophages, but not B cells, showed high uptake per cell (median fluorescence intensity, MFI) of both AlbiCpG and AlbiCSIINFEKL (Supplementary Fig. 23), with 15-20\% AlbiCpG ${ }^{+}$AlbiCSIINFEKL ${ }^{+}$DCs and macrophages (Fig. 3g), demonstrating efficient intracellular co-delivery of $\mathrm{CpG}$ and peptide Ag to LN APCs. Super-resolution imaging using instant SIM revealed that AlbiCSIINFEK ${ }_{(\text {FITC })} \mathrm{L}$ and AlbiCpGAlexa555 were co-localized in BMDCs within $2 \mathrm{~h}$, recapitulating efficient intracellular co-delivery of $\mathrm{CpG}$ and Ag via albumin/

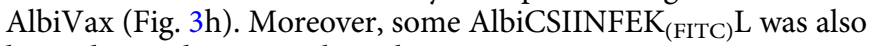
located in the cytosol. Relative to $\mathrm{CpG}+\mathrm{CSIINFEK}_{(\mathrm{FITC})} \mathrm{L}$,

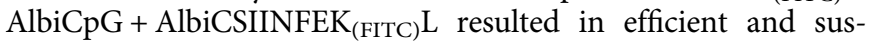
tained antigen presentation in BMDCs (Fig. 3i and Supplementary Fig. 24a). The intracellular co-delivery was likely mediated by binding of AlbiCpG and AlbiCSIINFEKL with separate albumin molecules, because Förster resonance energy transfer between AlbiCpG-Alexa555 and AlbiCSIINFEK (FITC) $_{\text {L }}$ was undetectable in the presence of albumin (Supplementary Fig. 24b). We hypothesize that albumin/AlbiVax would dissociate in the acidic endolysosome, as evidenced by 20 -fold weaker binding affinity of AlbiCpG with MSA at $\mathrm{pH} 5$ than at $\mathrm{pH} 7.4$ (Supplementary Fig. 24c). This dissociation will likely liberate AlbiVax, thereby preventing AlbiVax from trafficking out of APCs together with recycling albumin, and enhancing intracellular immunostimulation of AlbiCpG and cytosolic delivery of Ag.

AlbiVax elicited potent and durable T cell responses. Albumin/ AlbiCpG nanocomplexes were then studied for $\mathrm{T}$ cell responses with OVA. Compared with CpG + OVA, AlbiCpG + OVA (i.e., AlbiVax)-pulsed BMDCs markedly enhanced the proliferation of OT-1 CD8 ${ }^{+} \mathrm{T}$ cells (Supplementary Fig. 25a), suggesting that albumin/AlbiCpG promoted antigen cross presentation. We then immunized C57BL/6 mice with $2 \mathrm{nmol}$ AlbiCpG and $10 \mu \mathrm{g}$ OVA on day 0 and day 14 , and stained $\mathrm{CD}^{+} \mathrm{T}$ cells in peripheral blood using a $\mathrm{H}-2 \mathrm{~K}^{\mathrm{b}}$-SIINFEKL tetramer on day 21 (Fig. $4 \mathrm{a}$ and Supplementary Fig. 25b). The frequencies of tetramer ${ }^{+} \mathrm{CD}^{+} \mathrm{T}$ cells were $2.9 \pm 0.2 \%(n=5)$ induced by CpG + OVA, $3.8 \pm 0.2 \%(n=$ 5) by $\mathrm{CpG}+\mathrm{OVA}$ emulsified in benchmark IFA, and by contrast, $16.5 \pm 2.56 \%(n=7)$ by AlbiCpG + OVA $(p<0.01)$. No significant $\mathrm{T}$ cell responses were induced by control AlbiGpC + OVA, again indicating low immunogenicity of the MEB moiety. AlbiCpG + OVA increased the repertoires of polyfunctional IFN $\gamma$ ${ }^{+}, \mathrm{TNF}^{+}$, and IFN $\gamma^{+} \mathrm{TNF}^{+}$peripheral $\mathrm{CD}^{+} \mathrm{T}$ cells, thus potentiating the cytotoxicity of CTLs (Fig. $4 \mathrm{~b}$ and Supplementary Fig. 25c). AlbiCpG + OVA enhanced the serum titers of Agspecific IgG2a, which benefits cancer therapy (Supplementary Fig. 25d). Upon immunization with AlbiCpG + OVA, $16.7 \pm 3.2 \%$ $(n=5)$ of total peripheral $\mathrm{CD}^{+} \mathrm{T}$ cells expressed immune checkpoint PD-1 (Supplementary Fig. 25e, f). Among SIINK$\mathrm{FEKL}^{+} \mathrm{CD}^{+} \mathrm{T}$ cells, this frequency increased to $86.1 \pm 6.1 \%(n=$ 7), and the PD-1 MFI was fivefold higher than that on total CD8 ${ }^{+}$ T cells (Fig. 4c). The upregulated PD-1 expression upon AlbiVax elicited $\mathrm{T}$ cell activation suggests chronic antigen stimulation that eventually caused $\mathrm{T}$ cell exhaustion. Compared with total $\mathrm{CD} 8^{+}$ $\mathrm{T}$ cells, these differential phenotypic characteristics of Ag-specific $\mathrm{CD}^{+} \mathrm{T}$ cells indicate that Ag-specific $\mathrm{CD}^{+} \mathrm{T}$ cells exhibit a more exhausted state upon vaccination, in line with clinical observations $^{4,49}$. Given low spontaneous antitumor $\mathrm{T}$ cell responses in cancer patients and low to medium response rates of immune checkpoint blockade, the above observations provide the rationale to simultaneously elicit $\mathrm{T}$ cell responses and enhance the response rates to checkpoint inhibitors, such as anti-PD-1, for optimal therapy. A second boosting vaccination on day 28 further expanded the frequency of Ag-specific CD8 ${ }^{+}$CTLs to $21.0 \pm 2.5 \%$ $(n=7)$ on day 35 . This frequency remained $>5 \%$ by day 70 (Fig. 4d), indicating immune memory, which was further demonstrated by a large repertoire of SIINKFEKL ${ }^{+}$central memory $\mathrm{T}$ cells $\left(\mathrm{CD} 8^{+} \mathrm{CD} 44^{+} \mathrm{CD} 62 \mathrm{~L}^{\text {high }}\right)$ on day 70 (Fig. $4 \mathrm{e}$ and Supplementary Fig. 26). On day 71, immunized mice were s.c. challenged with $3 \times 10^{5}$ EG7.OVA cells. Marginal survival benefits were observed in mice vaccinated with CpG + OVA, despite early delay of tumor progression. IFA(CpG + OVA) moderately protected against challenge. By contrast, AlbiCpG + OVA significantly protected mice from challenge, with $71 \%(5 / 7)$ mice remaining tumor-free for more than 3 months (Fig. $4 \mathrm{f}, \mathrm{g}$ ). The surviving mice were re-challenged s.c. with $3 \times 10^{5}$ EG7.OVA cells 120 days after $1^{\circ}$ challenge, and $4 / 5$ mice survived the $2^{\circ}$ challenge for more than 6 months, again demonstrating durable $\mathrm{T}$ cell responses elicited by albumin/AlbiVax nanocomplexes. Of note, the binding of AlbiCpG with OVA $\left(K_{\mathrm{d}}=2.3 \mu \mathrm{M} ; R^{2}=0.96\right.$. Supplementary Fig. 25g) likely contributed to co-delivery of AlbiCpG and OVA to LNs and T cell responses.

We then investigated AlbiCpG + OVA for immunotherapy of established tumors. C57BL/6 mice were s.c. inoculated with $3 \times$ $10^{5}$ EG7.OVA, primed on day 6 post inoculation $\left(\sim 35 \mathrm{~mm}^{3}\right.$ tumors), and boosted on day 12 and day 18 ( $2 \mathrm{nmol} \mathrm{CpG}, 20 \mu \mathrm{g}$ OVA). While $\mathrm{CpG}+\mathrm{OVA}$ moderately retarded tumor progression, AlbiCpG + OVA markedly regressed tumors in all mice upon boosting (Fig. 4h). The eventual tumor recurrence likely resulted from immunoregulatory checkpoints, such as PD-1 on CD8 CTLs and PD-L1 on EG7.OVA cells (Supplementary Fig. 27a), and combination of AlbiCpG + OVA with anti-PD-1 would likely reduce tumor recurrence. While depletion of $\mathrm{CD} 4^{+}$ $\mathrm{T}$ cells or natural killer 1.1 (NK1.1) cells marginally affected the 
a

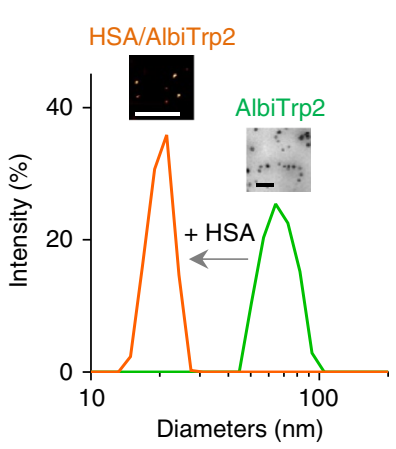

d

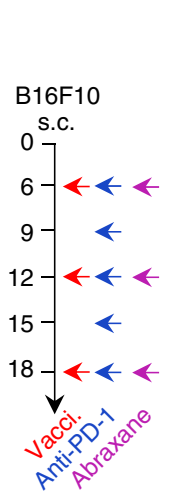

b
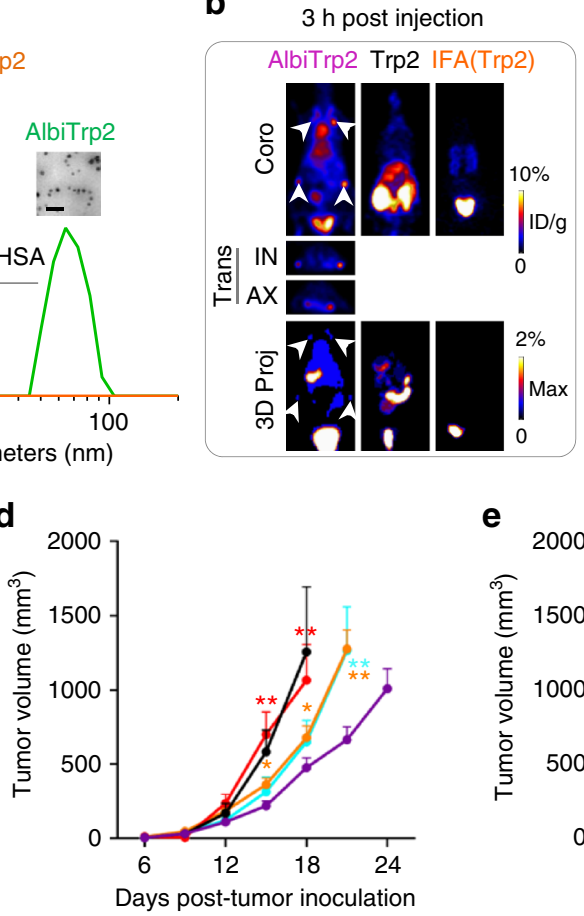

e

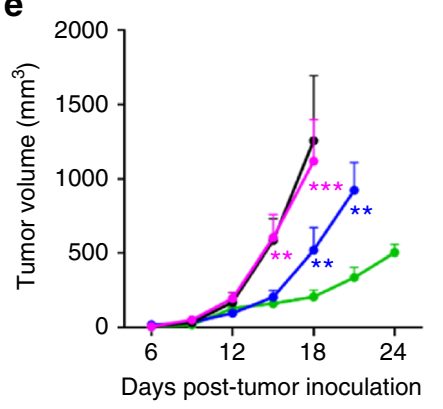

Time post injection (h)

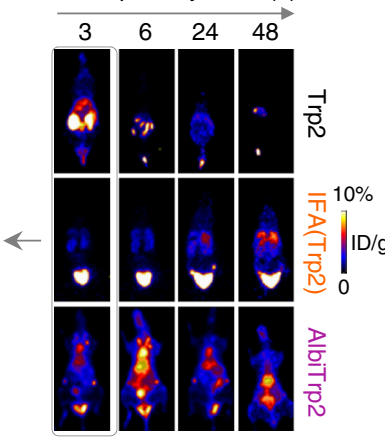

C

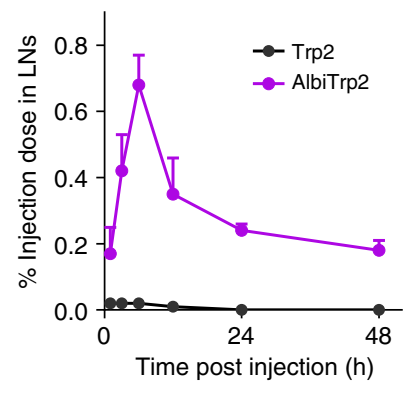

f

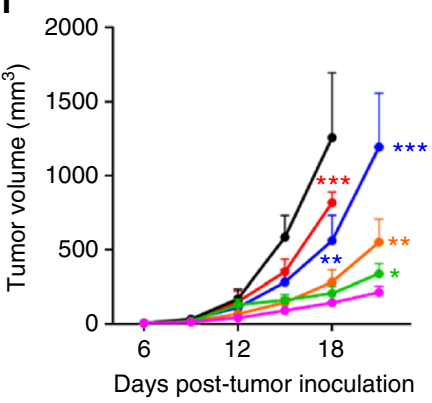

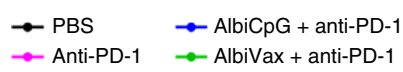

$\rightarrow$ PBS $\rightarrow$ Abraxane + anti-PD-1

$\rightarrow$ Abraxane $\rightarrow$ Abraxane + AlbiVax

$\rightarrow$ AlbiVax + anti-PD-1

$\rightarrow$ Abraxane + AlbiVax + anti-PD-1
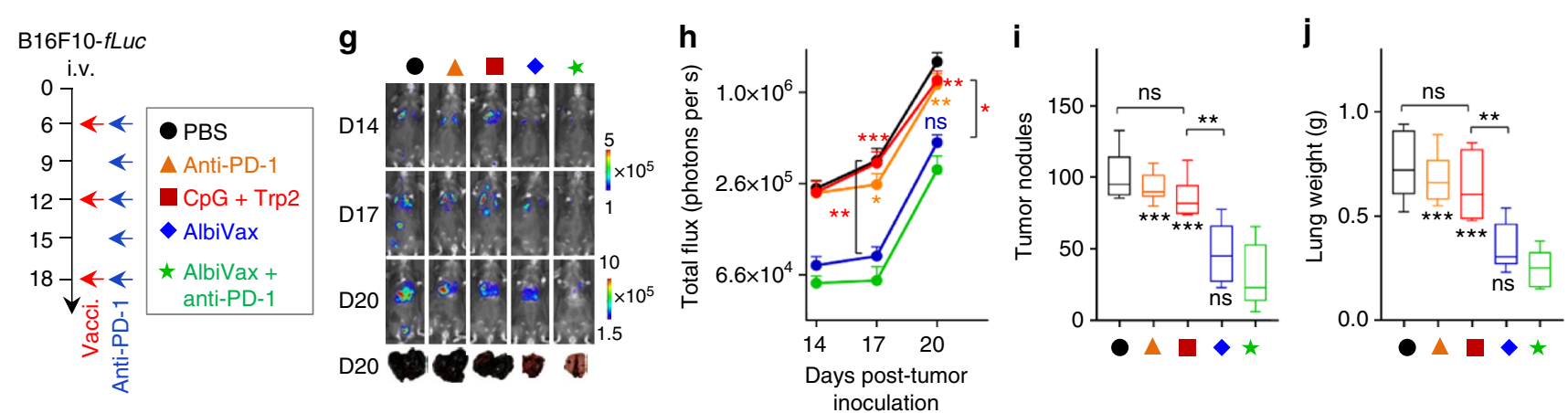

Fig. 5 Albumin/AlbiVax nanocomplexes for melanoma combination immunotherapy. a Transformation of AlbiTrp2 nanoparticles into albumin/AlbiTrp2 nanocomplexes in the presence of HSA (molar ratio of HSA: albumin=1:1). Insets: an AFM image (left) and a TEM image showing the corresponding nanoparticles. Scale bars: $200 \mathrm{~nm}$. b Representative coronal, transverse, and 3D projection of PET images at $3 \mathrm{~h}$ post injection (left), and representative coronal PET images at 3, 6, 24, and $48 \mathrm{~h}$ post s.c. injection of AlbiTrp2, free Trp2, and IFA(Trp2) at the tail base of FVB mice. (Dose: 4.4-5.5 Mbq.) c Quantification of albumin/AlbiTrp2 nanocomplexes and Trp2 in draining LNs (IN + AX). White arrows mark LNs. d-f B16F10 tumor growth after treatment with AlbiVax (d), double combination of albumin/AlbiVax nanocomplexes and anti-PD-1 (e), and triple combination of albumin/AlbiVax nanocomplexes, anti-PD-1, and Abraxane (f). C57BL/6 mice were s.c. inoculated with $3 \times 10^{5}$ B16F10 cells, treated with AlbiVax ( 2 nmol CpG equivalents $+20 \mu \mathrm{g}$ AlbiTrp2) (day 6, day 12, and day 18), anti-PD-1 every 3 days from day 6 for five times $\left(200 \mu \mathrm{g}\right.$ ), and Abraxane on day 6, day 12 , and day $18\left(20 \mathrm{mg} \mathrm{kg}{ }^{-1}\right)$. g-j C57BL/ 6 mice were i.v. injected with $1 \times 10^{5}$ B16F10-fLuc cells, treated with AlbiVax ( $2 \mathrm{nmol}$ CpG equivalents $+20 \mu \mathrm{g}$ AlbiTrp2) on day 6, day 12, and day 18 and anti-PD-1 (200 $\mu \mathrm{g})$ every 3 days from day 6 for six times. $\mathbf{g}$ Representative bioluminescence images on day 14, day 17, and day 20, and photographs of lungs on day 20. i Quantified bioluminescence intensities of lungs. i, j Numbers of tumor nodules (i) and lung weights (j) on day 20 . Data show mean \pm s.e. m. of two-three independent experiments. ${ }^{\star \star}{ }^{\star} p<0.001,{ }^{\star \star} p<0.01,{ }^{\star} p<0.05$, ns not significant $(p>0.05)$ by one-way ANOVA with Bonferroni post-test

therapeutic outcome of AlbiCpG + OVA, depletion of $\mathrm{CD}^{+}$ $\mathrm{T}$ cells nearly completely neutralized its efficacy, suggesting the central role of $\mathrm{CD}^{+}$CTLs in AlbiVax-based immunotherapy (Fig. 4h). To study the Ag specificity of immunotherapy, C57BL/6 mice were s.c. inoculated with $3 \times 10^{5} \mathrm{EL} 4$ cells $\left(\mathrm{OVA}^{-}\right)$on the left shoulder and $3 \times 10^{5}$ EG7.OVA on the right shoulder. Treatment with AlbiCpG + OVA again regressed 4/6 EG7.OVA tumors, whereas EL4 tumor progression was barely affected (Supplementary Fig. 27b, c), thus confirming the Ag specificity of AlbiVax-mediated immunotherapy. Again, AlbiCpG + OVA elevated frequency of peripheral SIINFEKL ${ }^{+} \mathrm{CD}^{+}$CTLs (Supplementary Fig. $27 \mathrm{~d}$ ). Moreover, no morbidity or toxicity was observed in AlbiVax-treated mice (Supplementary Fig. 27e).

Albumin/AlbiVax for combination melanoma immunotherapy. We then studied peptide-Ag-based AlbiVax for immunotherapy of melanoma, an aggressive skin tumor whose clinical outcome can be improved by potentiating antitumor immunity. A melanoma-associated subunit Ag, tyrosinase-related protein 2 
a

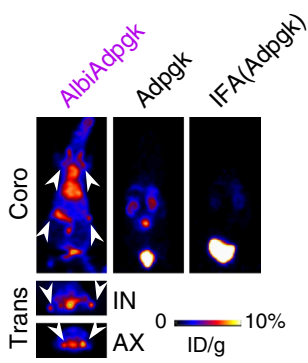

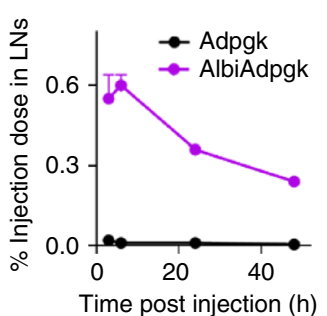

d

C

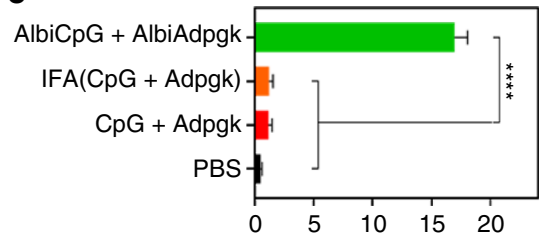

Percent of Adpgk-specific cells among live $\mathrm{CD}^{+} \mathrm{T}$ cells in PBMCs b

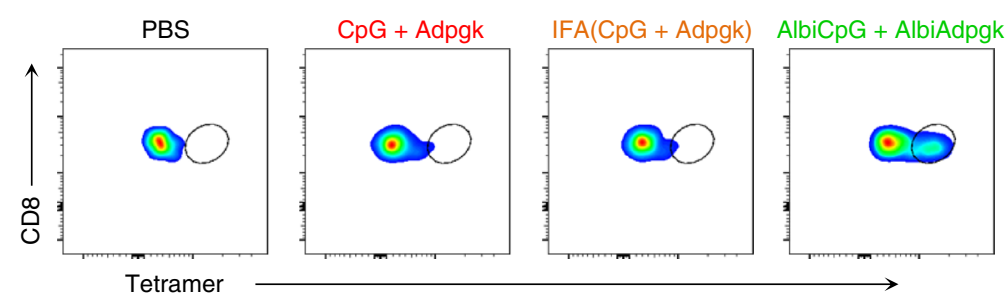

e
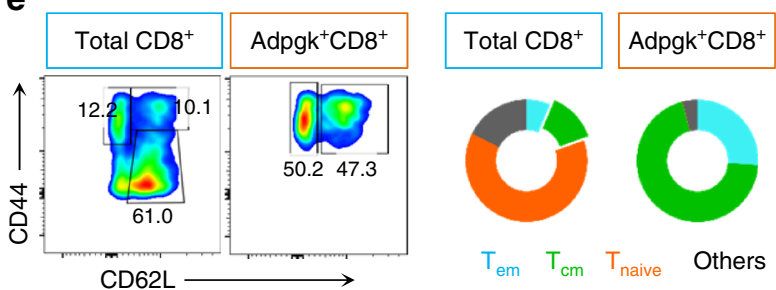

f

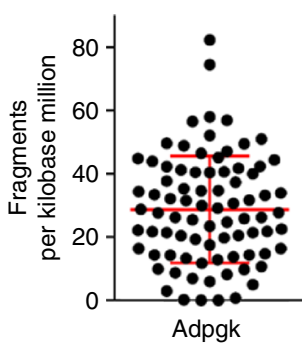

h

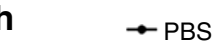

$\rightarrow$ AlbiVax

$\rightarrow$ AlbiVax (CD8 depl.)

$\rightarrow$ AlbiVax (CD4 depl.)

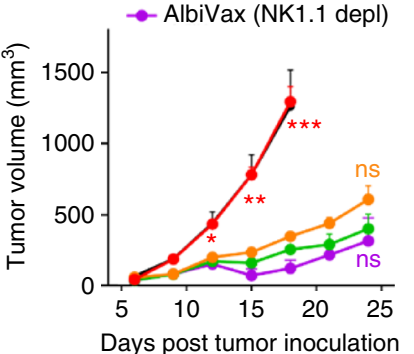

g

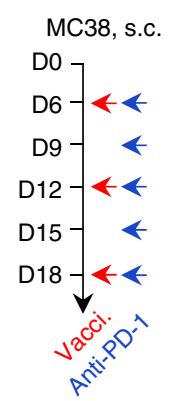

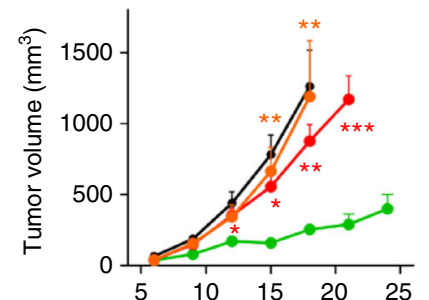

Days post-tumor inoculation
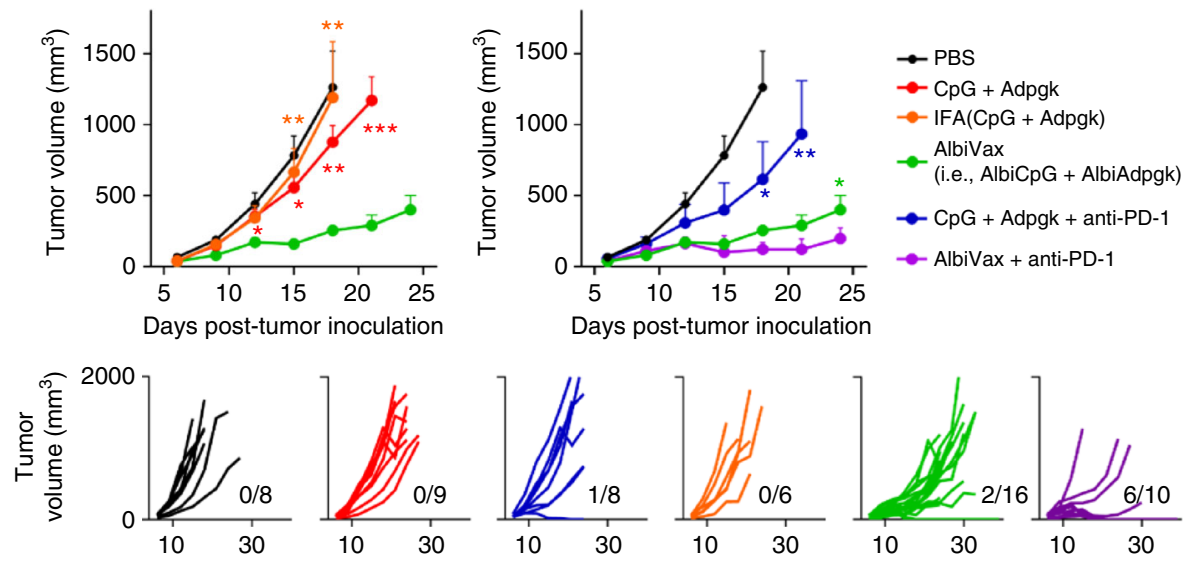

Days post-tumor inoculation
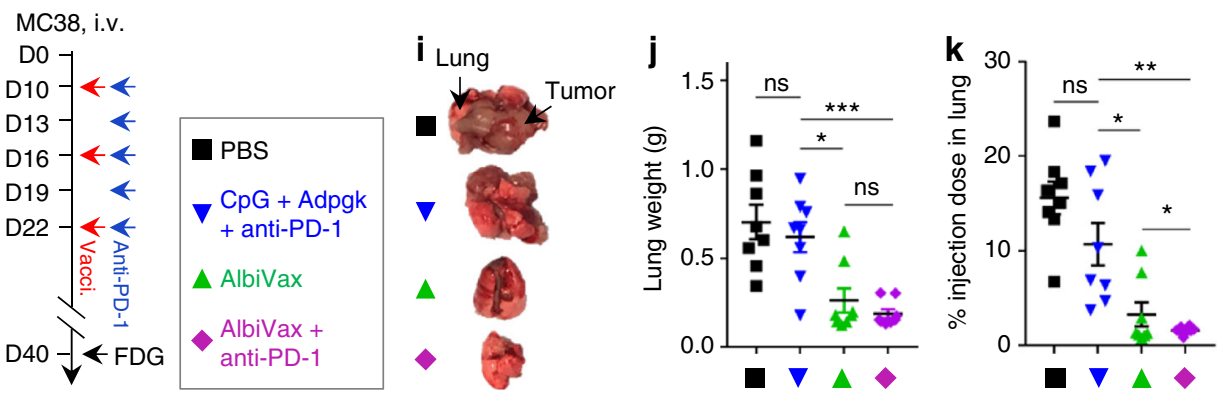

Fig. 6 Neoantigen-based albumin/AlbiVax nanocomplexes for personalized cancer immunotherapy. a Upper: representative coronal and transverse PET images at $6 \mathrm{~h}$ post injection, and lower: quantification of albumin/AlbiAdpgk nanocomplexes and Adpgk in IN and AX LNs of FVB mice ( $n=4)$. White arrows mark LNs. IFA(Adpgk) was undetectable in LNs. (Dose: 4.4-5.5 Mbq) b-e C57BL/6 mice $(n=5)$ were vaccinated with AlbiVax (2 nmol AlbiCpG +

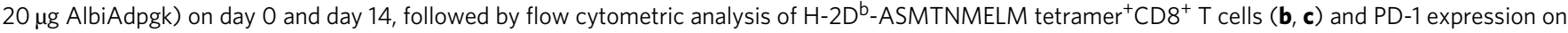
peripheral $\mathrm{CD}^{+} \mathrm{T}$ cells (d) on day 21, and $\mathrm{CD} 8^{+} \mathrm{T}$ cell central memory (CD62 $\mathrm{L}^{\text {high }} \mathrm{CD} 44^{+}$) on day 50 (e). f Exome-sequencing results verifying Adpgk variant in MC38 cells. g, h MC38 tumor growth after treatment with AlbiVax alone or in combination with anti-PD-1. C57BL/6 mice were s.c. inoculated with $3 \times 10^{5}$ MC38 cells, treated with AlbiVax ( 2 nmol AlbiCpG $+20 \mu$ g AlbiAdpgk) on day 6, day 12, and day 18), and with anti-PD-1 (200 $\mu$ g) every 3 days from day 6 for six times. $\mathbf{h}$ Depletion of $C D 8^{+} T$ cells, but not CD4 ${ }^{+}$T cells or NK cells abrogated the therapeutic efficacy of AlbiVax $(n=5)$. i-k Immunotherapy of lung metastatic MC38 tumor with AlbiVax alone or in combination with anti-PD-1. C57BL/ 6 mice were i.v. inoculated with $1 \times 10^{5}$ MC38 cells, treated with AlbiVax ( $2 \mathrm{nmol}$ AlbiCpG $+20 \mu \mathrm{g}$ AlbiAdpgk) on day 10, day 16, and day 22), and with anti-PD-1 (200 $\mu \mathrm{g})$ every 3 days from day 10 for six times. On day 40 , mice were injected with FDG tracer $(100 \mu \mathrm{Ci})$. Mice were killed and lungs and tumors were collected $(\mathbf{i})$, weighted $(\mathbf{j})$, and radioactivity measured by $\gamma$-counting $(\mathbf{k})$. Data show mean \pm s.e.m. of two-three independent experiments. ${ }^{\star \star *} p<0.001,{ }^{\star \star} p<0.01,{ }^{\star} p<0.05$, ns: not significant $(p>0.05)$ by one-way ANOVA with Bonferroni post-test 
(Trp2), was used in this study. Trp2 modified with an N-terminal cysteine was conjugated with MEB. Hydrophilic MEB increased the water solubility of MEB-Trp2 conjugate relative to $\operatorname{Trp} 2$, resulting in self-assembled amphiphilic MEB-Trp2 nanoparticles in aqueous solution (Fig. 5a). MEB-Trp2 was tightly bound to albumin $\left(K_{\mathrm{d}}=0.79 \mu \mathrm{M} . R^{2}=0.88\right)$, which drove the transformation from MEB-Trp2 (AlbiTrp2) amphiphilic nanoparticles to albumin/AlbiTrp2 nanocomplexes and recovered MEB fluorescence (Fig. 5a and Supplementary Fig. 28). Trp2 delivery was quantitatively imaged by PET in FVB mice s.c. injected with ${ }^{64} \mathrm{Cu}$-labeled AlbiTrp2 at the tail base. While Trp2 was rapidly disseminated systemically, leaving no detectable Trp2 in draining LNs, albumin/AlbiTrp2 nanocomplexes were efficiently delivered to LNs with 91-fold larger AUC than Trp2 over 3 days (Fig. 5b, c). By contrast, IFA-emulsified Trp2 dramatically retained Trp2 at the injection sites, which might not only sequestrate, but also disarm and delete Ag-specific CD8 ${ }^{+}$CTLs.

Syngeneic C57BL/6 mice vaccinated with AlbiVax (i.e., AlbiCpG + AlbiTrp2) significantly resisted a s.c. challenge of $3 \times 10^{5} \mathrm{~B} 16 \mathrm{~F} 10$ cells, in contrast to marginal protection by $\mathrm{CpG}+$ Trp2 or IFA(CpG + Trp2) (Supplementary Fig. 29a). In B16F10 tumor-bearing C57BL/6 mice, PET verified that both AlbiCpG and AlbiTrp2 were efficiently delivered to LNs, except for tumor draining LNs due to damaged lymphatic drainage in tumors (Supplementary Fig. 29b-e). For immunotherapy, C57BL/6 mice s.c. inoculated with $3 \times 10^{5} \mathrm{~B} 16 \mathrm{~F} 10$ cells were treated with AlbiVax ( $2 \mathrm{nmol} \mathrm{CpG}$ equivalent, $20 \mu \mathrm{g}$ AlbiTrp2) on day 6, day 12 , and day 18 post inoculation. While none of $\mathrm{CpG}+\operatorname{Trp} 2$, AlbiCpG + Trp2, or IFA(CpG + Trp2) significantly inhibited tumor progression, albumin/AlbiVax nanocomplexes significantly prohibited tumor growth (Fig. 5d). Given the high expression of PD-L1 on B16F10 tumor cells (Supplementary Fig. 29f), a combination of anti-PD-1 and albumin/AlbiVax nanocomplexes enhanced the therapeutic efficacy (Fig. 5e). A triple combination of anti-PD-1, albumin/AlbiVax nanocomplexes, and chemotherapeutic Abraxane that enhances tumor immunity ${ }^{50,51}$, further inhibited the tumor progression (Fig. 5f). No tumors were eradicated, but we envision that enhanced therapeutic efficacy can be achieved using multi-epitope albumin/ AlbiVax to induce a broader spectrum of antitumor $\mathrm{T}$ cell responses, or by combining with more synergistic therapeutics ${ }^{16,32}$. Taking advantage of the systemic $T$ cell responses induced by albumin/AlbiVax, we then investigated immunotherapy of metastatic melanoma, a primary death cause of melanoma patients. A lung metastatic B16F10 model was established by intravenous (i.v.) injection of $1 \times 10^{5}$ B16F10-fLuc cells into $\mathrm{C} 57 \mathrm{BL} / 6$ mice. When lung metastases were established, mice were again treated with AlbiVax on day 6, day 12, and day 18, and anti-PD-1 on day 6 , day 9 , day 12 , day 15 , and day 18 post-cell injection. Lung metastatic B16F10-fLuc burdens were monitored by bioluminescence imaging (Fig. 5g, h). Neither CpG + Trp2 nor anti-PD-1 alone significantly inhibited tumor progression, whereas albumin/AlbiVax inhibited the deposition and progression of lung metastases (Fig. 5i, j). Again, combining albumin/ AlbiVax nanocomplexes and anti-PD-1 further potentiated the therapeutic efficacy.

Neoantigen-based albumin/AlbiVax for tumor immunotherapy. We next explored albumin/AlbiVax nanocomplexes for neoantigen-based personalized tumor immunotherapy. We used Adpgk, a neoantigen [ASMTN $(\mathrm{R} \rightarrow \mathrm{M}) \mathrm{ELM}$ ] in MC38 tumors ${ }^{6}$. The conjugation of hydrophilic MEB improved the water solubility of MEB-Adpgk, and entailed MEB-Adpgk to strongly bind to albumin $\left(K_{\mathrm{d}}=1.1 \mu \mathrm{M} . \quad R^{2}=0.96\right)$ and form amphiphilic nanoparticles in aqueous solution (Supplementary Fig. 30a, b).
Using ${ }^{64} \mathrm{Cu}$-labeled NMEB-Adpgk (denoted MEB-Adpgk as AlbiAdpgk), PET demonstrated efficient LN delivery of albumin/ AlbiAdpgk, and the AUC in IN and AX LNs was 43-fold higher than that of Adpgk within 2 days (Fig. 6a and Supplementary Fig. 30c, d). IFA(Adpgk) was again trapped in the injection sites, and despite the particulate formulation of AlbiAdpgk, significantly less AlbiAdpgk was trapped at the injection sites than IFA(Adpgk) (Supplementary Fig. 30e), which is particularly significant for subunit Ag-based vaccination to prevent the dysfunction of functional $\mathrm{CD}^{+}$CTLs. Immunization of $\mathrm{C} 57 \mathrm{BL} / 6$ mice with AlbiVax (AlbiCpG + AlbiAdpgk) on day 0 and day 14 elicited 14.1- and 13.6-fold greater frequency of peripheral Adpgk ${ }^{+} \mathrm{CD}^{+}$CTLs than CpG + Adpgk and IFA(CpG + Adpgk), respectively, as stained using a $\mathrm{H}-2 \mathrm{D}^{\mathrm{b}}$-ASMTNMELM tetramer (Fig. 6b, c). Albumin/AlbiVax upregulated PD-1 expression on peripheral $\mathrm{CD}^{+}$CTLs, especially $\mathrm{Adpgk}^{+} \mathrm{CD}^{+}$CTLs (Fig. 6d), suggesting that $\mathrm{T}$ cell responses were accompanied with $\mathrm{T}$ cell exhaustion that justified PD-1 blockade for optimal therapy. Further, albumin/AlbiVax induced central T cell memory for over 50 days (Fig. 6e). Immunizing C57BL/6 mice with AlbiVax on day 0 and day 14 markedly retarded MC38 tumor progression after s.c. challenge with $3 \times 10^{5}$ MC38 cells on day 30 (Supplementary Fig. 30f).

To study albumin/AlbiVax nanocomplexes for personalized immunotherapy of MC38 tumor, the Adpgk variant was verified in MC38 cells by exome sequencing (Fig. $6 \mathrm{f}$ ). A total of $3 \times 10^{5}$ MC38 cells were s.c. inoculated in C57BL/6 mice, and treatment was initiated on day 6 when tumors were established $\left(\sim 30 \mathrm{~mm}^{3}\right)$. In contrast to the moderate inhibition of tumor progression by IFA(CpG + Adpgk), albumin/AlbiVax inhibited tumor progression significantly more effectively (Fig. $6 \mathrm{~g}$ ). Most tumors failed to regress, in part due to immune checkpoints $\mathrm{PD}-1$ on $\mathrm{CD}^{+}$CTLs (Fig. 6c) and PD-L1 on MC38 cells (Supplementary Fig. 30g). Thus, by combining anti-PD-1 with AlbiVax, CD8 ${ }^{+}$CTLs reinvigoration markedly increased the response rate and led to complete regression of $6 / 10$ tumors for 4 months (Fig. $6 \mathrm{~g}$ and Supplementary Fig. 30h). These tumor-free mice resisted a rechallenge with MC38 cells, again suggesting long-term immune memory. Lymphocyte depletion demonstrated that $\mathrm{CD}^{+} \mathrm{T}$ cells, but not CD4 T cells or NK1.1 cells, were vital to AlbiVaxmediated immunotherapy (Fig. 6h). Lung is a primary destination of colon cancer metastases. Lung metastatic-like tumors were established by i.v. injection of $1 \times 10^{5} \mathrm{MC} 38$ cells into C57BL/6 mice. From day 10 post-tumor inoculation, mice were treated with AlbiVax every 6 days for three times and anti-PD-1 every 3 days for six times. On day 40, the metabolic activity of lung metastatic-like tumors was studied using ${ }^{18} \mathrm{~F}$-fluorodeoxyglucose (FDG), as shown by PET-CT of PBS-treated mice (Supplemental Fig. 30i). Organs of interest were resected, weighted, and radioactivity was measured by $\gamma$ counting. Tumor burdens (Fig. 6i, j) and FDG radioactivity (Fig. 6k and Supplemental Fig. 30j) of lung metastatic-like tumors suggest that albumin/ AlbiVax significantly reduced tumor progression, and combination with anti-PD-1 further potentiated the therapeutic efficacy (Fig. 6k).

\section{Discussion}

Safe and robust $\mathrm{T}$ cell vaccines would hold great promise for combination cancer immunotherapy. Locally administered vaccines must provide efficient delivery to LNs for effective antitumor immunomodulation. Nanovaccines are a promising approach for efficient vaccine delivery to LNs, and quite a few have been developed preclinically, yet the clinical translation of these nanoformulas has been hindered by limitations in their safety, suitability for reproducible large-scale manufacturing, 
and in vivo integrity. We chose albumin, one of the most successful carriers of nanomedicines in the clinic, to deliver anticancer molecular vaccines in this study. We exploited the ability of clinically safe EB to bind with endogenous albumin and to concentrate within LNs, and we engineered AlbiVax by conjugating EB derivatives with molecular vaccines to efficiently codeliver adjuvant and peptide Ags into LNs for combination cancer immunotherapy. In contrast to synthetic nanomedicines, such as Abraxane, albumin/AlbiVax nanocomplexes were assembled from endogenous albumin and exogenous molecular vaccines in vivo. This approach is intrinsically attractive for multiple reasons. First, EB-based AlbiVax has shown a good safety profile. EB had been considerably practiced in the clinic, and currently no side effects of EB derivatives were observed in mice or in two clinical trials of systemically or locally injected EB derivatives in hundreds of patients and healthy volunteers ${ }^{36,39}$. Moreover, rhesus monkeys survived $25 \mathrm{mg} \mathrm{kg}^{-1}$ systemically injected $\mathrm{EB}^{40}$. Though the safety of albumin/AlbiVax nanocomplexes remains to be systematically determined, a low dose is sufficient for potent immunization $\left(0.4 \mathrm{mg} \mathrm{kg}^{-1}\right.$ MEB equivalent, $<0.2 \%$ of dose used in the above monkeys). Moreover, local administration and reduced systemic dissemination of AlbiVax further mitigate toxicity risk. Second, chemically defined AlbiVax are amenable to relatively easy large-scale manufacturing, formulation, and quality control, and they offer long shelf-life. Large-scale synthesis of AlbiVax via a biocompatible thiolmaleimide conjugation is expected to be generally applicable for molecular vaccines. Third, AlbiVax harnesses endogenous albumin to assemble nanocomplexes, thus avoiding the complications involved with safety, large-scale production and quality control of synthetic nanomaterials. Fourth, albumin/AlbiVax nanocomplexes have a comprehensive and sophisticated delivery mechanism: efficient delivery to LNs by lymphatic drainage, efficient intracellular delivery to APCs by endocytosis, and conditional intracellular vaccine release by albumin/AlbiVax dissociation in acidic endolysosomes. Efficient intracellular vaccine delivery is essential for Ag cross presentation and robust $\mathrm{T}$ cell responses. Internalized albumin/AlbiVax nanocomplexes in APCs is dissociated in the acidic endolysosome, probably due to loosened binding, thereby liberating AlbiVax for intracellular immunomodulation and preventing AlbiVax from efflux during albumin recycling. Fifth, EB-based AlbiVax is expected to be widely applicable for therapeutics ranging from peptides, nucleic acids, lipids, polysaccharides, to small molecules. AlbiVax was synthesized via conjugation of MEB and a thiol that can be easily modified for many chemical or biologic therapeutics; alternatively, we also developed EB derivatives with alkyne and amine that can be used for AlbiVax conjugation. Recombinant albumin-drug conjugates, by contrast, can only be applied to protein or peptide vaccines, which are inefficient at intracellular vaccines release that is however pivotal for potent immunomodulation; moreover, using endogenous albumin would obviate problems resulting from contamination during biologics production or immunogenic responses of exogenous albumin conjugates. Covalent conjugation of exogenous therapeutics to the cysteine-34 position of endogenous albumin, though prolong circulation, is limited again by inefficient intracellular vaccine release and nonspecific conjugation with other cysteine-accessible biomolecules including apolipoprotein B-100. Compared with albumin-binding peptides and hydrophobic lipids, hydrophilic EB derivatives may improve drug formulation especially for hydrophobic drugs, and avoid off-target interactions with surrounding biomolecules or cell membranes ${ }^{52}$. PEG can improve the aqueous solubility, but also adds up cost and increases the risk of allergy against $\mathrm{PEG}^{53}$, especially when an immunostimulatory adjuvant is co-administered.
We systematically and quantitatively studied vaccine delivery and revealed the spatiotemporal intranodal and intracellular behaviors of AlbiVax by multiscale imaging, including PET in small animals, light sheet fluorescence microscopy in whole LNs, and super-resolution fluorescence imaging in single APCs. Interestingly, super-resolution imaging allowed us to reveal that AlbiCpG was located primarily on APC endolysosome membrane likely due to binding to TLR9 on the membranes, and we observed substantial co-localization of AlbiCpG and AlbiAg in APCs as a result of albumin-mediated endocytosis. These observations may provide insight for the underlying mechanism of AlbiVax-mediated immunomodulation. Albumin/AlbiVax nanocomplexes co-delivered adjuvants and Ags to APCs and enabled sustained Ag presentation on APCs. In mice, albumin/ AlbiVax nanocomplexes dramatically enhanced the potency and durability of $\mathrm{T}$ cell responses, and significantly inhibited tumor progression or eradicated tumors in multiple primary or metastatic tumors. There are over 1000 clinical trials of combination cancer immunotherapy involving vaccines currently under enthusiastic pursuit ${ }^{54}$. The principle that albumin/AlbiVax nanocomplexes are amenable for combination therapy with immune checkpoint inhibitors and chemotherapy is encouraging preclinical evidence supporting AlbiVax-based combination immunotherapy.

Albumin/AlbiVax nanocomplexes are readily applicable to neoantigen vaccination for personalized tumor immunotherapy. Although tumor immunotherapy benefits from high loads of tumor-specific mutations and high frequencies of neoantigenspecific CTLs ${ }^{7,9,55-57}$, natural neoantigen-specific CD8 ${ }^{+}$CTLs are often rare (e.g., $0.002-0.4 \%$ in melanoma, which already has the second highest tumor mutation loads ${ }^{58,59}$ ), likely due to low clonal neoantigen burden, inefficient antigen processing and cross presentation, and immunosuppression. Exogenous neoantigen vaccines delivered by albumin/AlbiVax nanocomplexes can potentiate neoantigen-specific immunity and potentiate tumor therapy. Further, combining checkpoint inhibitors with albumin/ AlbiVax nanocomplexes can further unleash CTL activity and enhance therapeutic efficacy. While it currently takes a relatively long time to identify and manufacture neoantigen-based AlbiVax, patients can be treated with other regimens before AlbiVax.

In summary, by in vivo assembly with endogenous albumin, albumin/AlbiVax nanocomplexes represent a widely applicable $\mathrm{T}$ cell vaccine that, as quantitatively determined, is efficiently delivered into LNs, ameliorates side effects, induces potent and durable $\mathrm{T}$ cell responses, and as part of combination immunotherapy inhibits or eradicates established tumors.

\section{Methods}

DNA synthesis. DNA was synthesized at a $1 \mu$ mole scale of solid phase synthesis on an ABI 392 DNA synthesizer (Applied Biosystems) using materials purchased from Glen Research (Sterling, VA) or Chemgenes (Wilmington, MA). DNA was deprotected accordingly to the manufacturer's instructions, and purified by reverse phase HPLC (Dionex Ultimate 3000, Thermo Fisher Scientific, Waltham, MA). Dimethoxytrityl (DMT) protecting group on DNA was removed using $0.5 \mathrm{M}$ acetic acid. DNA was desalted and quantified on a Genesys $10 \mathrm{~s}$ UV-Vis spectrometer (Thermo Fisher Scientific, Waltham, MA). Fluorophores, thiol, or HEG were modified following the manufacturer's instructions. All DNAs, including HEG linkers, had phosphorothioate backbone. DNA modified with Alexa488 or Alexa555 were from Integrated DNA Technology (Coralville, IA).

AlbiVax synthesis. CpG1826 (TCCATGACGTTCCTGACGTT) and GpC control (TCCATGAGCTTCCTGAGCTT) were used. AlbiCpG or derivatives were synthesized using MEB and terminal thiol-modified CpG. For AlbiCpG screening, four CpG derivatives with $0,1,2$, and 3 hexaethyloxy-glycol (HEG) units between MEB (confirmed by NMR analysis) ${ }^{35}$ and $\mathrm{CpG}$ were used. Thiol-modified DNA was pretreated with DTT $(0.1 \mathrm{M})$ in PBS $\left(37^{\circ} \mathrm{C}, 1 \mathrm{~h}\right)$ to cleave the dithiol bond, fol lowed by desalting using a NAP5 column in sodium ascorbate buffer $(0.1 \%)$ to remove DTT and the thiol-appending small fragment cleaved from DNA. The resulting DNA $(200 \mathrm{nmol})$ was mixed with MEB $(1 \mathrm{mg})$ in $2 \mathrm{~mL}$ sodium ascorbate 
buffer $(0.1 \%)$ in PBS and reacted at room temperature for $30 \mathrm{~min}$. The resulting product was purified again using a NAP5 column to remove excess MEB, and quantified by UV absorbance at $260 \mathrm{~nm}$ (where MEB has negligible absorption compared with $\mathrm{CpG}$ ) or by weighing lyophilized products in the case of synthesis at a relatively large scale.

MEB-antigen conjugates were synthesized using MEB and antigens (CSIINFEKL, Trp2, and Adpgk) that were modified with $\mathrm{N}$-terminal cysteine. (Trp2: CSVYDFFVWL; Adpgk: CGIPVHLELASMTNMELMSSIVHQQVFPT.) In a typical reaction, MEB $(10 \mathrm{mg})$ dissolved in water $(1 \mathrm{~mL})$ was added to peptide $(10 \mathrm{mg})$ dissolved in DMF $(2 \mathrm{~mL})$ dropwise. The reaction mixture was agitated at room temperature for at least 1 day. MEB-antigen conjugates were purified using a C18 column on HPLC.

AlbiVax was verified using liquid chromatography-electrospray ionization-tandem mass spectrometry (LC-ESI-MS).

AFM and TEM. The sizes and morphologies of nanomaterials were characterized using transmission electron microscopy (TEM) and atomic force microscopy (AFM). TEM samples of AlbiVax or a mixture of AlbiVax and HSA were prepared by depositing samples $(10 \mu \mathrm{L})$ onto a carbon-coated copper grid. For AFM, samples $(10 \mu \mathrm{L})$ were casted on freshly peeled mica substrate, followed by drying, rinsing, and dehumidifying. AFM was carried out in tapping mode in air on a PicoForce Multimode AFM (Bruker, CA) equipped with a Nanoscope ${ }^{\circledR} \mathrm{V}$ controller, a type E scanner head, and a sharpened TESP-SS (Bruker, CA) AFM cantilever. AFM images were then analyzed by Nanoscope Software (version 7.3-8.15, Bruker, CA).

Molecular docking and prediction of albumin/MEB binding. The HSA model was constructed using SwissPdb Viewer ${ }^{60,61}$ based on the X-ray complex structure of indoxyl sulfate with HSA (Protein Data Bank (PDB) ID code 2BXH) ${ }^{33}$, with the ligand and water molecules removed. The stereochemical restraints were obtained from the CHARMM molecular mechanics force field ${ }^{62}$. The model was then calculated via cycles of geometry optimization and molecular dynamics simulation using NAMD $^{63}$, with energy-minimized HSA structure. The stereochemical quality was analyzed using PROCHECK ${ }^{64}$.

Automated molecular docking was conducted using AutoDock version 4.2.6 ${ }^{65}$. The Lamarckian genetic algorithm (LGA) was applied to model the binding of MEB with HSA. For molecular docking, LGA describes the relationship between a ligand and a protein by the translation, orientation, and conformation of the ligand. The final solution provides a ligand conformation finely tuned to the protein ${ }^{66}$. The docking area (active site) of HSA was defined using the AutoDock module AutoGrid. The grid site was constrained to a $28.5 \AA$ cubic space, including the entire binding site of HSA and providing sufficient space for translational and rotational walk of the ligand. The LGA was applied to search the conformational and orientational space of MEB while keeping the HSA structure rigid. Default parameters were used, except the maximum energy evaluations of $1.5 \times 10^{6}$. With 10 independent runs, a maximum of $2.7 \times 10^{4}$ genetic algorithm operations were generated on a single population of 50 individuals. Further, binding free energy and affinity constant were calculated, and the docked complex was selected based on matching interaction energies with geometric quality. Further energy minimization and geometric optimization were performed on the select complex until no more conflicts among the ligand and HSA.

A score function at binding free energy was derived and adopted in AutoDock ${ }^{65}$, based on the traditional molecular force field model of interaction energy. The restriction of internal rotors, global rotation, and translation were modeled depending on the calculated torsion angles of the ligand. The total binding free energy was empirically calibrated, using 30 structurally known protein-ligand complexes with experimentally determined binding constants. The score function is sufficient to rank the ligands on level of binding affinity and free energy ( $\Delta G_{\text {binding }}$ values), as well as the inhibition constant $\left(K_{\mathrm{i}}\right)$. With this approach, we then calculated the binding free energy and inhibition constant.

Photophysics of MEB-DNA. Photophysics of MEB-DNA was studied in terms of fluorescence spectrometry, intensity, and lifetime, in the absence or presence of albumin. All measurements were conducted on a fluorometer (Jobin Yvon, HORIBA) using excitation and emission parameters specific for MEB.

Kinetic binding assay by BLI. The kinetic binding of AlbiVax with albumin was measured by BLI on an Octet Red96 system (fortéBio) using streptavidin-coated biosensors (fortéBio). Assays were carried out with agitation in solid black 96-well plates (Geiger Bio-One) in seven steps: 1, baseline (1x PBS), time: $60 \mathrm{~s}$; 2, loading (Biotin-labeled albumin, $1 \mu \mathrm{g} \mathrm{m}^{-1}$ ), time: $600 \mathrm{~s} ; 3$, baseline (1× PBS), time: $60 \mathrm{~s} ; 4$, quenching $\left(1 \mu \mathrm{g} \mathrm{mL} \mathrm{L}^{-1}\right.$ Biocytin (Thermo Scientific)), time: $180 \mathrm{~s}$; 5 , baseline (1× PBS), time: $60 \mathrm{~s}$; 6, association, time: $600 \mathrm{~s}$; 7, dissociation, time: $600 \mathrm{~s}$. Nonspecific binding was performed by measuring the binding of albumin-loaded biosensor to buffer alone and blank biosensor to analytes. Data analysis and curve fitting were performed using Octet Analysis software 7.0. Binding data were fitted with a binding model of 1:1 ligand interaction using GraphPad Prism 7 (La Jolla, CA).

Animal studies. All animal work was conducted in appliance to the NIH Guide for the Care and Use of Animals under protocols approved by the NIH Clinical Center
Animal Care and Use Committee. Female C57BL/6j mice (6-8 weeks. The Jackson Laboratory) were used for fluorescence imaging of AlbiVax delivery, immunology analysis, and tumor therapy studies. Female FVB mice (6-8 weeks) were used in all PET studies of AlbiVax delivery in tumor-free mice as well as toxicity studies of AlbiCpG.

PET imaging and biodistribution of AlbiVax. AlbiVax was radiolabeled with ${ }^{64} \mathrm{Cu}$ for PET. To label ${ }^{64} \mathrm{Cu}$ via chelation, a NOTA-functionalized MEB (NMEB) was synthesized and conjugated with vaccines ${ }^{44}$. Specifically, ${ }^{64} \mathrm{CuCl}_{2}$ was converted to ${ }^{64} \mathrm{Cu}$-acetate by adding $0.5 \mathrm{~mL}$ of $0.4 \mathrm{M} \mathrm{NH}_{4} \mathrm{OAc}$ solution (pH 5.6) to 20 $\mu \mathrm{L}{ }^{64} \mathrm{CuCl}_{2}{ }^{64} \mathrm{Cu}$-acetate solution $(0.1 \mathrm{~mL} ; 3-4 \mathrm{mCi})$ was added into a solution of $100 \mu \mathrm{g}$ of CpG or NMEB-CpG or derivatives in water, or NMEB-Adpgk/NMEBTrp2 in DMSO $\left(10 \mathrm{mg} \mathrm{mL}^{-1}\right)$. The reaction was shaken for $0.5 \mathrm{~h}$ at $37^{\circ} \mathrm{C}$. Thereafter, the radiochemical purity was determined using iTLC plates (Fisher Scientific), developed in $0.1 \mathrm{M}$ citric acid (pH 5). Crude reaction of $\mathrm{CpG}$ or NMEB$\mathrm{CpG}$ derivatives were purified on a Nap5 column (GE Healthcare) and the desired product was eluted in saline. NMEB-Adpgk and NMEB-Trp2 were purified on a C18 Sep-Pak (BOND-ELUT $100 \mathrm{mg}$, Varian); then NMEB peptides were eluted from the cartridge using $70 \%$ ethanol and 30\% PBS.

Female FVB mice (6-8 weeks) were used in all PET imaging studies in naive mice. C57BL/6 mice (6-8 weeks) were used in PET imaging of tumor-bearing mice Mice were anesthetized using isoflurane/ $\mathrm{O}^{2}\left(2 \% \mathrm{v} \mathrm{v}^{-1}\right)$ before injection. Anesthetized mice were injected s.c. at the base of tail with ${ }^{64} \mathrm{Cu}$-labeled vaccines (4.44-5.55 MBq/120-150 $\mu \mathrm{Ci}$ each mouse) in PBS $(100 \mu \mathrm{L})$. At indicated time points post injection, mice were scanned on an Inveon DPET scanner (Siemens Medical Solutions, Malvern, PA). PET images were reconstructed without correction for attenuation or scattering using a three-dimensional ordered subsets expectation maximization algorithm. ASI Pro $\mathrm{VM}^{\mathrm{TM}}$ software was used for image analysis. Regions of interest (ROI) were drawn on LNs to calculate the \% $\mathrm{ID} / \mathrm{g}$.

The above mice were killed at specified time points. Organs and blood were collected and wet weighed. The collected organs and blood, together with a series of standard solutions, were measured for ${ }^{64} \mathrm{Cu}$ radioactivity on a gamma counter (Wallac Wizard 1480, PerkinElmer). The radioactivity of organs and blood was converted to calculate the percentages of the injected dose (\%ID) in organs of interest and the percentages of the injected dose per gram of tissue $(\% \mathrm{ID} / \mathrm{g})$.

LN clearing and light sheet fluorescence imaging of LNs. Draining LNs of vaccinated C57Bl/6 mice were cleared using a modified PACT (passive CLARITY technique $)^{46}$. Particularly, female C57BL/6 mice (6-8 weeks) were injected with AlbiCpG ( $5 \mathrm{nmol}$ each mouse) s.c. at the base of the tail. After $24 \mathrm{~h}$, transcardiac perfusion was conduct with $6 \mathrm{~mL}$ PBS and then $6 \mathrm{~mL} 4 \%$ paraformaldehyde (PFA). PFA-fixed LNs were then isolated and incubated at $4{ }^{\circ} \mathrm{C}$ overnight in hydrogel monomer solution in PBS containing $4 \%$ acrylamide and $0.25 \%$ photoinitiator 2,20-Azobis[2-(2-imidazolin-2-yl) propane] dihydrochloride (VA-044, Wako Chemicals USA). LNs infused with hydrogel monomer were degassed with nitrogen for $5 \mathrm{~min}$ and then incubated for $2 \mathrm{~h}$ at $37^{\circ} \mathrm{C}$ to initiate tissue-hydrogel crosslinking. LNs were then washed with PBS to remove excess hydrogel. For tissue clearing, LN-hydrogel matrices were incubated in $8 \%$ SDS in $0.1 \mathrm{M}$ PBS (pH 7.5) for 2 days at $37^{\circ} \mathrm{C}$ with gentle shaking. LNs were cleared well and were then washed with PBS for four times in 1 day.

To image cleared LNs, LNs were mounted in refractive index matching solution (RIMS) with a refractive index (RI) of 1.46 , for 1 day before imaging. RIMS was prepared by adding $40 \mathrm{~g}$ Histodenz (Sigma D2158) in $30 \mathrm{~mL} 0.02 \mathrm{M} \mathrm{PB} \mathrm{(pH} \mathrm{7.5)}$ with $0.1 \%$ Tween- 20 and $0.01 \%$ sodium azide. To mount LNs for imaging, LNs were embedded in $2 \%$ agarose in a $500-\mathrm{mL}$ syringe. Once agarose was gelled in the syringe, the LN-gel matrices were push partially out of syringe and immersed in RIMS for 1 day to allow RIMS to penetrate the agarose gel and create a homogeneous imaging media.

Light sheet 3D imaging of LNs were performed on a Lightsheet Z.1 light sheet microscope (Carl Zeiss Microscopy, LLC).

Cell lines and cell culture. EL4 and EG7.OVA cells were from Dr Joshua Farber's laboratory at NIAID, NIH. EL4 cells were cultured in RPMI medium with $2 \mathrm{mM} \mathrm{L-}$ glutamine, 10\% heat-inactivated FBS, and 1\% penicillin and streptomycin; EG7. OVA cells were cultured in RPMI medium with $2 \mathrm{mM}$ L-glutamine, $10 \%$ heatinactivated FBS, and $0.4 \mathrm{mg} \mathrm{mL}^{-1}$ G418. MC38 cells were from Dr Robert Seder Laboratory at NIH Vaccine Research Center. RAW264.7 cells (from ATCC), B16F10 cells (from ATCC), and MC38 cells were cultured in DMEM medium with $10 \%$ heat-inactivated FBS and 1\% penicillin and streptomycin. DC2.4 cells were from Dr Jonathan W. Yewdell Lab at NIAID, and cultured in RPMI medium with $2 \mathrm{mM}$ L-glutamine, $10 \%$ heat-inactivated FBS, and $1 \%$ penicillin and streptomycin Cells were grown in a humidified atmosphere $\left(5 \% \mathrm{CO}_{2}, 37^{\circ} \mathrm{C}\right)$. All cells were tested to be free of mycoplasma.

Antibodies for flow cytometry and tissue staining. Antibodies were from $\mathrm{BD}$ Bioscience, eBioscience, and BioLegend. The following antibodies are listed in the order of antigen, fluorophore, clone, sources, catalog number, and dilution times for the final concentrations. CD11c, FITC, N418, Biolegend, 117306, 1600; CD11c, PE, HL3, BD, 553802, 800; F4/80, APC, BM8, eBioscience, 17-4801-82, 800; CD3, 
PerCP Cy5.5, 17A2, Biolegend, 100218, 800; B220, BV421, RA3-6B2, Biolegend, 103240, 800; B220, Al647, RA3-6B2, Biolegend, 103226, 1600; CD8a, APC-Cy7, 536.7, Biolegend, 100714, 800; CD8a, APC, 53-6.7, BD, 553035, 800; PD-1, BV421, 29F.1A12, Biolegend, 135221, 800; PD-L1, BV421, 10F.9G2, Biolegend, 124315, 800; IFN-gamma, PE, XMG1.2, BD, 554412, 100; IFN-gamma, FITC, XMG1.2, eBioscience, 11-7311-82, 100; TNF-alpha, FITC, MP6-XT22, BD, 560659, 100; TNF-alpha, APC, MP6-XT22, eBioscience, 560658, 100; CD62L, FITC, MEL-14, BD, 553150; CD44, PE-Cy5, IM7, BD, 561861. Antibodies were used according to the manufacturer's instructions, including antibody dilution. Flow cytometry was conducted on a BD LSRFortessa X-50 flow cytometer at the Core Flow Cytometry Facility of Vaccine Research Center.

In vitro cell uptake of AlbiCpG in APC. In vitro cell uptake was first studied using confocal laser scanning microscopy and flow cytometry. FITC-labeled AlbiCpG were incubated with RAW264.7 cells, DC2.4 cells, or BMDCs, and stained with Lysotracker Red DND-99 (Life Technologies, Carlsbad, CA) and $10 \mu \mathrm{gL}^{-1}$ Hoechst 33342 (Life Technologies, Carlsbad, CA) for $0.5 \mathrm{~h}$ in a cell culture incubator at $37^{\circ} \mathrm{C}$, prior to observation. Cells were then washed with Dulbecco's PBS for three times before imaging on a Zeiss LSM 780 confocal microscope (Chesterfield, VA). Alternatively, flow cytometry was used to study the cell uptake using a BD Beckman Coulter flow cytometer (Brea, CA) or BD Accuri C6 flow cytometer (San Jose, CA). For flow cytometric analysis, RAW264.7 cells, or DC2.4 cells, or BMDC cells were seeded into 24-well plate, and 1 day later, cells were treated with AlbiCpG for a specified time, followed by flow cytometric analysis. Flow cytometry results were analyzed by using FlowJo V10 software.

In vitro cell uptake of AlbiCpG into APCs by $\gamma$-counting. The cell uptake of AlbiCpG was also studied using ${ }^{64} \mathrm{Cu}$-labeled AlbiCpG. RAW264.7 cells or DC2.4 cells $\left(2 \times 10^{5}\right.$ cells per well) were seeded onto a 12 -well plate, and 1 day later, cells were treated with AlbiCpG ( 2 uCi per well) for a specified time, followed by washing with Dulbecco's PBS, treating cells with $\mathrm{NaOH}$ solution, and collecting the resulting solution to measure the radioactivity on a $\gamma$ counter.

Super-resolution imaging of intracellular AlbiCpG in APCs. Cultured cells were treated with AlbiCpG for $2 \mathrm{~h}$ and washed with PBS. Endolysosome was stained with LysoTracker Green (Invitrogen, Carlsbad, CA) according to the manufacturer's instruction. Cells were immersed in HEPES buffer ( $\mathrm{pH}$ 7.4) during imaging. Super-resolution imaging was conducted on an instant linear structured illumination microscope (instant SIM) (built in Dr Hari Shroff Laboratory) ${ }^{41}$. On iSIM, Alexa488-labeled AlbiCpG (Ex: $488 \mathrm{~nm}$ ) and LysoTracker Red DND-99 (Ex: $561 \mathrm{~nm}$ ) was used for imaging for a long duration. Images were deconvolved and analyzed using home-built software ${ }^{41}$. Alternatively, confocal microscopy with deconvolution was conducted on a Leica SP8 workstation (Leica Microsystem, IL). On the Leica SP8 workstation, MEB fluorescence was monitored to image AlbiCpG (Ex: $561 \mathrm{~nm}$; Em: 600-670 nm), confirming that there was minimal overlap in fluorescence between LysoTracker Green and MEB. Raw imaging results were deconvolved and analyzed on the Leica SP8 workstation.

Proinflammatory factors. The concentrations of proinflammatory factors (TNF $\alpha$, IL-6, and IL-12p40) were measured using ELISA (Thermo Fisher Scientific) according to the manufacturers' instructions ${ }^{13}$. Cell culture medium from cells treated in vitro was collected at the specified time points post treatment. Sera of treated mice were collected at the specified time points post treatment. Medium or sera were diluted according to the manufacturers' instructions.

Splenomegaly. Female FVB mice (6-8 weeks. $n=5)$ were injected with unconjugated $\mathrm{CpG}$ or AlbiCpG (5 nmol each mouse unless denoted otherwise). After 6 days, unless denoted otherwise, mice were weighted, spleens were collected, and the splenomegaly was assessed by determining the ratio of (spleen weight)/(mouse weight).

Costimulatory factors on APCs. CD80 and CD86 were profiled on cell surfaces of RAW264.7 macrophages and BMDCs by flow cytometry. After treatment, cells were dissociated using non-enzymatic dissociation buffer. Dissociated cells were washed with PBS and resuspended in PBS for staining. To analyze costimulatory factors on LN-resident APCs, draining inguinal LNs were collected from mice treated with $\mathrm{CpG}$ or AlbiCpG (s.c. at the tail base), followed by physical tissue dissociation using sharp needles. Cells were pipetted for a few times to further dissociate cells, and filtered using a $40-\mu \mathrm{m}$ cell strainer to remove tissue debris. Cells were washed twice using PBS, counted, and stained in PBS with dye-labeled antibodies at $4^{\circ} \mathrm{C}$ for $30 \mathrm{~min}$, followed by washing with PBS and flow cytometric analysis. Flow cytometry results were analyzed by using FlowJo V10 software.

In vitro antigen cross presentation. $\mathrm{CD} 11 \mathrm{c}^{+} \mathrm{BMDCs}$ were isolated from female C57BL/6 mice (6-8 weeks) using positive selection beads (Miltenyi), and treated overnight with $(\mathrm{OVA}+\mathrm{AlbiCpG})$ vs. $(\mathrm{OVA}+\mathrm{CpG})$. OT- $1 \mathrm{CD}^{+} \mathrm{T}$ cells were isolated, labeled with CFSE, and added to co-culture with the above BMDCs. After
2 days, cells were stained with DAPI and anti-CD8-APC, and $\mathrm{T}$ cell proliferation was determined by divided viable $\left(\mathrm{DAPI}^{-}\right) \mathrm{CD}^{+}$cells in flow cytometry.

AlbiVax uptake in LN-residing APCs. Dye-labeled AlbiVax or MSA (CpG: 2 nmol equivalent; antigen: $20 \mathrm{nmol}$ equivalent; MSA: $20 \mathrm{nmol}$ ) were s.c. injected at the base of the tail of female C57BL/6j mice (6-8 weeks. $n=6-8)$. LNs were isolated 1-3 days post injection. LNs were mechanically disrupted, pipetted, and filtered using $40-\mu \mathrm{m}$ cell strainers. Cells were blocked with anti-CD16/CD32 in FCS buffer for $10 \mathrm{~min}$, followed by staining for B cells $\left(\mathrm{B} 220^{+}\right)$, DCs $\left(\mathrm{CD} 11 \mathrm{c}^{+}\right)$, and macrophages $\left(\mathrm{F} 4 / 80^{+}\right)$, and analyzed by flow cytometry for AlbiVax or MSA fluorescence signal. Data were analyzed using FlowJo V10.

Anti-OVA IgG1. Female C57BL/6 mice (6-8 weeks. $n=5$ ) were vaccinated with AlbiCpG and OVA on day 0 and day 14. Peripheral blood was collected from vaccinated mice on day 21 . Blood cells were precipitated by centrifugation and removed, and the resulting sera were enriched to measure anti-OVA IgG, IgG1, IgG2a, and IgM. Sera were diluted 1000 times for ELISA analysis.

Tetramer staining and PD-1 expression on PBMCs. Mouse peripheral CD8 ${ }^{+}$ $\mathrm{T}$ cells were stained for antigen-specific tetramer as described previously ${ }^{15}$. PEconjugated $\mathrm{H}-2 \mathrm{~K}^{\mathrm{b}}$-SIINFEKL tetramer and $\mathrm{H}-2 \mathrm{D}^{\mathrm{b}}$-ASMTNMELM tetramer (manufactured at the NIH Tetramer Core Facility) were used for tetramer staining of OVA-vaccinated mice and Adpgk-vaccinated mice, respectively. Briefly, mice were treated with AlbiVax on day 0 and day 14. Blood was collected from the treated mice on day 21 . Blood cells were enriched by centrifugation. Red blood cells were lysed using ACK lysis buffer for $10 \mathrm{~min}$ at room temperature. Blood clots were removed using a filter. Cells were washed twice in PBS and cells were stained using Live/Dead Fixable Green Dead Cell Stain Kit for $10 \mathrm{~min}$ at room temperature. Staining was quenched and cells were washed with FCS buffer (PBS buffer with $0.1 \%$ FBS). Cells were then blocked with anti-CD16/CD32 for $10 \mathrm{~min}$, followed by adding a dye-labeled antibody cocktail (CD3-PerCP Cy5.5, CD8-APC-Cy7, Tetramer-PE, PD-1-BV421) and staining at room temperature for $30 \mathrm{~min}$. Cells were then washed, and $100 \mu \mathrm{L}$ Cytofix was added into each well to resuspend cells, and cells were fixed at $4{ }^{\circ} \mathrm{C}$ for $20 \mathrm{~min}$. Cells were then washed with Perm/Wash buffer, and resuspended for flow cytometric analysis. Flow cytometry was conducted on a BD LSRFortessa X-50 flow cytometer. Data were analyzed using FlowJo V10 version.

Intracellular cytokine staining in T cells. Female C57BL/6 mice (6-8 weeks. $n=$ 5) were vaccinated with AlbiVax on day 0 and day 14. Peripheral blood was collected from vaccinated mice on day 21 . Red blood cells were removed by incubating with ACK lysis buffer for $5 \mathrm{~min}$ and centrifugation for $10 \mathrm{~min}$, and the obtained lymphocytes were transferred into U-bottom 96-well plate in $200 \mu \mathrm{L} \mathrm{T}$ cell culture media were: RPMI 1640 supplemented with $10 \% \mathrm{FBS}, 100 \mathrm{U} \mathrm{mL}^{-1}$ penn/ strep, $50 \mu \mathrm{M} \beta$-mercaptoethanol, 1x MEM non-essential amino acid solution, and $1 \mathrm{mM}$ sodium pyruvate. Lymphocytes were pulsed with antigen epitopes $(20 \mu \mathrm{g} \mathrm{mL}$ ${ }^{-1}$ ) for $2 \mathrm{~h}$, followed by adding GolgePlug Protein Transport Inhibitor containing brefeldin A. Cells were then placed in a culture incubator for $4 \mathrm{~h}$. Next, cells were incubated with anti-CD16/CD32 for $10 \mathrm{~min}$ at room temperature, then the cells were stained with anti-CD8-APC-Cy7 and DAPI for $20 \mathrm{~min}$ at room temperature. Cells were washed and subsequently fixed using Cytofix (BD Biosciences), washed, and permeabilized in $200 \mu \mathrm{L}$ Cytoperm solution (BD Biosciences) according to the manufacturer's instructions. Cells were washed using Perm/Wash Buffer (BD Biosciences). Permeabilized cells were then stained using anti-IFN $\gamma$-PE and antiTNF $\alpha$-FITC according to the manufactor's guidance. Stained cells were then washed and resuspended for flow cytometric analysis. Flow cytometry was conducted on a BD LSRFortessa X-50 flow cytometer. Data were analyzed using FlowJo V10 version.

Immune memory. Female C57BL/6 mice (6-8 weeks. $n=6-8)$ were vaccinated as described above. Peripheral blood was collected to analyze antigen-specific CD8 $\mathrm{T}$ cells and memory $\mathrm{T}$ cells. Immune memory was analyzed by flow cytometric analysis of peripheral lymph node homing receptor, CD62L, and adhesion molecule, CD44. Briefly, red blood cells were lysed using ACK lysis buffer, and blood cells were then collected by centrifugation and washing with FCS buffer (PBS buffer with $0.1 \%$ FBS). Cells were then blocked with anti-CD16/CD32 in FCS buffer for $10 \mathrm{~min}$, followed by adding dye-labeled antibody cocktail (CD8-APC-Cy7, CD44PE-Cy5, CD62L-FITC, and dead cell-staining DAPI) and staining at room temperature for $30 \mathrm{~min}$. Cells were then washed, and $100 \mu \mathrm{L}$ Cytofix was added into each well to resuspend cells. Cells were allowed for fixation at $4{ }^{\circ} \mathrm{C}$ for $20 \mathrm{~min}$. Cells were then washed with Perm/Wash buffer, and resuspended for flow cytometric analysis. Central memory $\mathrm{CD} 8^{+} \mathrm{T}$ cells were analyzed as $\mathrm{CD} 44^{\mathrm{hi}} \mathrm{CD} 62 \mathrm{~L}^{\mathrm{hi}} \mathrm{CD} 8^{+}$ $\mathrm{T}$ cells; effector memory $\mathrm{CD}^{+} \mathrm{T}$ cells had variable to low levels of CD62L and high CD44 levels; and naive CD8 ${ }^{+} \mathrm{T}$ cells had high levels of CD62L and low to intermediate levels of CD44. Flow cytometry was conducted on a BD LSRFortessa X-50 flow cytometer. Data were analyzed using FlowJo V10 version.

$1^{\circ}$ tumor challenge was conducted by s.c. inoculation of tumor cells $\left(3 \times 10^{5}\right)$ on the right shoulder. Tumor sizes were monitored every 3 days thereafter. If applicable, $2^{\circ}$ tumor challenge was conducted by s.c. inoculation of tumor cells 
$\left(1 \times 10^{6}\right)$ on the right flank of mice that survived the $1^{\circ}$ tumor challenge. Tumor sizes were again monitored every 3 days thereafter. Mice were killed if any dimension of the tumor was close to or already exceeded $2 \mathrm{~cm}$. Tumor volume was calculated using the following formula:

$$
\text { Volume }=\left(\text { length } \times \text { width }^{2}\right) / 2
$$

Results were analyzed using GraphPad Prism 7 (La Jolla, CA).

Plasmid transfection of tumor cells. B16F10 and MC38 cells were transfected with plasmid pcDNA3.1 (+)-Neo-fLuc for firefly luciferase expression. Briefly, 70\% confluent cells were transfected using Lipofectamine 2000 (Invitrogen) according to the manufacturer's instructions. Two days after transfection, cells were treated with Geneticin $\left(0.4 \mathrm{mg} \mathrm{mL}^{-1}\right)$ to screen cells that stably express firefly luciferase for a total of 4 weeks. The resulting cells were confirmed for firefly luciferase expression using bioluminescence imaging. Cells that expressed firefly luciferase were further cultured in medium containing Geneticin $\left(0.4 \mathrm{mg} \mathrm{mL}^{-1}\right)$.

Tumor immunotherapy. For primary tumor models, female C57BL/6 mice (6-8 weeks. The Jackson Laboratory. $n=6-8$ ) were s.c. inoculated with EL4 cells, or EG7.OVA cells, B16F10 cells, MC38 cells on the shoulder. Tumor growth was monitored by caliper measurement. Mice were killedd when the maximal dimension of tumor reached about $2 \mathrm{~cm}$. For a lung metastatic melanoma model, female C57BL/6 mice (6-8 weeks) were i.v. injected with B16F10-fLuc cells or MC38 cells $\left(1 \times 10^{5}\right.$ per mouse).

To study antigen specificity of immunotherapy, $2 \times 10^{5}$ EL4 lymphoblastoma cells and $2 \times 10^{5}$ EG7.OVA (OVA expressing) were respectively inoculated on to the left and right shoulders of C57BL/6 mice (6-8 weeks). Four groups of mice (6-7 mice per group) were respectively vaccinated with (1) PBS, (2) CpG and OVA, (3) AlbiGpC and OVA, and (4) AlbiCpG and OVA (doses: $2 \mathrm{nmol} \mathrm{CpG} \mathrm{equivalents,}$ $20 \mu \mathrm{g}$ OVA), by s.c. injection in $50 \mu \mathrm{L}$ volume at the base of the tail on day 3 and day 9 post-tumor inoculation. Tumor sizes and mouse weights were monitored every 3 days. Mice were killed on day 19 when one dimension of most EL4 tumors was close to or already exceeded $2 \mathrm{~cm}$. The tumor volume was calculated and analyzed as described above.

A combination of immunotherapy (AlbiVax and anti-PD-1, Clone RMP1-14, Bio X cell) and chemotherapy (Abraxane) for melanoma treatment was studied in both a primary melanoma model and a lung metastatic melanoma model. In primary tumor model, $3 \times 10^{5} \mathrm{~B} 16 \mathrm{~F} 10$ cells in $100 \mu \mathrm{L}$ PBS were s.c. injected into the right shoulder of C57BL/6 mice. On day 6 post-tumor inoculation, when established tumors were palpable, mice were randomly divided into six groups (6-10 mice per group) for treatment. The dose is $1.4 \mathrm{nmol} \mathrm{CpG} \mathrm{equivalents,} 20 \mu \mathrm{g}$ Trp2 or AbTrp2, $200 \mu \mathrm{g}$ anti-PD-1 (Bio X Cell, Inc., NH), and $20 \mathrm{mg} \mathrm{kg}^{-1}$ Abraxane (Celgene, MD). Vaccines were injected s.c. at the base of tail, anti-PD-1 was injected intraperitoneally, and Abraxane was injected i.v. Maintenance treatment was performed at the following doses: anti-PD-1 every 3 days for a total of five times, vaccines every 6 days for a total of three times, and Abraxane every 6 days for a total of three times. Tumor sizes and mouse weights were monitored every 3 days. Mice were killed when one dimension of a tumor exceeded $2 \mathrm{~cm}$ or ulceration developed. The tumor volume was calculated and analyzed as described above. Results from two independent studies were pooled together. Results were analyzed using GraphPad Prism 7 (La Jolla, CA).

In the lung metastatic B16F10 study, $1 \times 10^{5} \mathrm{~B} 16 \mathrm{~F} 10-f L u c$ cells were injected i.v. into C57BL/6 mice. On day 6 when lung metastasis is established, treatment was initiated (vaccine: $2 \mathrm{nmol} \mathrm{CpG} \mathrm{equivalents,} 20 \mu \mathrm{g} \mathrm{Ag}$, every 6 days; anti-PD-1: 200 $\mu \mathrm{g}$, every 3 days). The growth of luciferase transgenic tumors was monitored by bioluminescence imaging after i.p. injection of an aqueous solution of D-luciferin $\left(0.1 \mathrm{ml}, 30 \mathrm{mg} \mathrm{mL}^{-1}\right.$, GoldBio, St. Louis, MO) on an IVIS Lumina (Caliper Life Sciences). Bioluminescence in regions of interest (ROI) were quantified as total flux. Mice were killed if they developed severe morbidity. Mice were all killed on day 20 post inoculation, and lungs were collected to enumerate the counts of lung metastatic nodules and measure lung weights.

In lung metastatic-like MC38 tumor studies, $1 \times 10^{5}$ MC38 cells were injected i.v. into C57BL/6 mice. On day 10 when lung metastasis is established, treatment was initiated (vaccine: $2 \mathrm{nmol} C \mathrm{CpG}$ equivalents, $20 \mu \mathrm{g} \mathrm{Ag}$, every 6 days; anti-PD-1: $200 \mu \mathrm{g}$, every 3 days). Mice were killed if they developed severe morbidity. At the end of study, mice were i.p. injected with FDG (3.7 Mbq). Representative mice were scanned by PET/CT on a nanoScan system (Mediso), and all mice were killed, and lungs collected to measure lung weights and radioactivity by $\gamma$-counting.

In lymphocyte depletion, female C57BL/6 mice (6-8 weeks) were s.c. inoculated with EG7.OVA or MC38 cells $\left(3 \times 10^{5}\right)$ on the right shoulder. On day 6 when tumors were established, mice were divided into five groups to have comparable tumor volumes $(n=5)$. Five groups of mice were respectively vaccinated with PBS in group (1), and AlbiVax ( 2 nmol CpG equivalents and $20 \mu \mathrm{g}$ OVA or AlbiAdpgk) in groups (2-5), by s.c. injection in $50 \mu \mathrm{L}$ PBS at the base of the tail on day 6,12 , and 18 post-tumor inoculation. Meanwhile, on days $6,9,12,15$, and 18 post-tumor inoculation, mice in groups (2-5) were also intraperitoneally injected with PBS in group (2), anti-CD4 in group (3), anti-CD8 in group (4), and anti-NK1.1 in group (5) (antibody dose: $200 \mu \mathrm{g}$ per mouse). Tumor sizes and mouse weights were monitored every 3 days. Mice were killed when any tumor dimension was close to or already exceeded $2 \mathrm{~cm}$. The tumor volume was calculated and analyzed as described above.

Data availability. The authors declare that all the data related with this study are available within the paper or can be obtained from the authors on request.

Received: 19 September 2017 Accepted: 13 November 2017 Published online: 05 December 2017

\section{References}

1. Rosenberg, S. A. Raising the bar: the curative potential of human cancer immunotherapy. Sci. Transl. Med. 4, 127ps128 (2012).

2. Hubbell, J. A., Thomas, S. N. \& Swartz, M. A. Materials engineering for immunomodulation. Nature 462, 449-460 (2009).

3. Irvine, D. J., Hanson, M. C., Rakhra, K. \& Tokatlian, T. Synthetic nanoparticles for vaccines and immunotherapy. Chem. Rev. 115, 11109-11146 (2015).

4. Gros, A. et al. PD-1 identifies the patient-specific $\mathrm{CD}^{+}$tumor-reactive repertoire infiltrating human tumors. J. Clin. Invest. 124, 2246-2259 (2014)

5. Kreiter, S. et al. Mutant MHC class II epitopes drive therapeutic immune responses to cancer. Nature 520, 692-696 (2015).

6. Yadav, M. et al. Predicting immunogenic tumour mutations by combining mass spectrometry and exome sequencing. Nature 515, 572-576 (2014).

7. McGranahan, N. et al. Clonal neoantigens elicit $\mathrm{T}$ cell immunoreactivity and sensitivity to immune checkpoint blockade. Science 351, 1463-1469 (2016).

8. Rizvi, N. A. et al. Mutational landscape determines sensitivity to PD-1 blockade in non-small cell lung cancer. Science 348, 124-128 (2015).

9. Schumacher, T. N. \& Schreiber, R. D. Neoantigens in cancer immunotherapy. Science 348, 69-74 (2015).

10. Krieg, A. M. Therapeutic potential of Toll-like receptor 9 activation. Nat. Rev. Drug Discov. 5, 471-484 (2006).

11. Klinman, D. M. Immunotherapeutic uses of CpG oligodeoxynucleotides. Nat. Rev. Immunol. 4, 249-258 (2004).

12. Hailemichael, Y. et al. Persistent antigen at vaccination sites induces tumorspecific CD8+ T cell sequestration, dysfunction and deletion. Nat. Med. 19, 465-472 (2013).

13. Zhu, G. et al. DNA-inorganic hybrid nanovaccine for cancer immunotherapy. Nanoscale 8, 6684-6692 (2016).

14. Kim, J. et al. Injectable, spontaneously assembling, inorganic scaffolds modulate immune cells in vivo and increase vaccine efficacy. Nat. Biotechnol. 33, 64-72 (2015).

15. Lynn, G. M. et al. In vivo characterization of the physicochemical properties of polymer-linked TLR agonists that enhance vaccine immunogenicity. Nat. Biotechnol. 33, 1201-1210 (2015).

16. Kuai, R., Ochyl, L. J., Bahjat, K. S., Schwendeman, A. \& Moon, J. J. Designer vaccine nanodiscs for personalized cancer immunotherapy. Nat. Mater. 16, 489-496 (2017).

17. Zhu, G., Zhang, F., Ni, Q., Niu, G. \& Chen, X. Efficient nanovaccine delivery in cancer immunotherapy. ACS Nano 11, 2387-2392 (2017).

18. Chen, H., Zhang, W., Zhu, G., Xie, J. \& Chen, X. Rethinking cancer nanotheranostics. Nat. Rev. Mater. 2, 17024 (2017).

19. Kratz, F. Albumin as a drug carrier: design of prodrugs, drug conjugates and nanoparticles. J. Control Release 132, 171-183 (2008).

20. Mitragotri, S., Burke, P. A. \& Langer, R. Overcoming the challenges in administering biopharmaceuticals: formulation and delivery strategies. Nat. Rev. Drug Discov. 13, 655-672 (2014).

21. Liu, H. et al. Structure-based programming of lymph-node targeting in molecular vaccines. Nature 507, 519-522 (2014).

22. Balboni, P. G. et al. Activity of albumin conjugates of 5-fluorodeoxyuridine and cytosine arabinoside on poxviruses as a lysosomotropic approach to antiviral chemotherapy. Nature 264, 181-183 (1976).

23. Sand, K. M. et al. Unraveling the interaction between FcRn and albumin: opportunities for design of albumin-based therapeutics. Front. Immunol. 5, 682 (2014).

24. Yardley, D. A. nab-Paclitaxel mechanisms of action and delivery. J. Control Release 170, 365-372 (2013).

25. Zhu, E. F. et al. Synergistic innate and adaptive immune response to combination immunotherapy with anti-tumor antigen antibodies and extended serum half-life IL-2. Cancer Cell 27, 489-501 (2015).

26. Moyer, T. J., Zmolek, A. C. \& Irvine, D. J. Beyond antigens and adjuvants: formulating future vaccines. J. Clin. Invest. 126, 799-808 (2016).

27. Larsen, M. T. \& Kuhlmann, M. Albumin-based drug delivery: harnessing nature to cure disease. Mol. Cell Ther. 4, 3 (2016).

28. Staak, JanO., Jianyi Wang, D. C., Sung, Cynthia. \& Andrew, A. Raubitschek radiolabeled IL-2-human serum albumin fusion protein (Albuleukin ${ }^{\mathrm{TM}}$ ) as a potential new reagent for radioimmunoimaging/therapy of Hodgkin's lymphoma. Blood 104, 4633 (2004). 
29. Trevaskis, N. L., Kaminskas, L. M. \& Porter, C. J. From sewer to saviour targeting the lymphatic system to promote drug exposure and activity. Nat. Rev. Drug Discov. 14, 781-803 (2015).

30. Zorzi, A., Middendorp, S. J., Wilbs, J. \& Deyle, K. Acylated heptapeptide binds albumin with high affinity and application as tag furnishes long-acting peptides. Nat. Commun. 8, 16092 (2017).

31. Sjölander, A., Nygren, P., Ståhl, S. \& Berzins, K. The serum albumin-binding region of streptococcal protein G: a bacterial fusion partner with carrier-related properties. J. Immunol. Methods 201, 115-123 (1997).

32. Moynihan, K. D. et al. Eradication of large established tumors in mice by combination immunotherapy that engages innate and adaptive immune responses. Nat. Med. 22, 1402-1410 (2016).

33. Ghuman, J. et al. Structural basis of the drug-binding specificity of human serum albumin. J. Mol. Biol. 353, 38-52 (2005).

34. Niu, G. et al. In vivo labeling of serum albumin for PET. J. Nucl. Med. $\mathbf{5 5}$ 1150-1156 (2014).

35. Wang, Y. et al. In vivo albumin labeling and lymphatic imaging. Proc. Natl Acad. Sci. USA 112, 208-213 (2015).

36. Zhang, J. et al. Clinical translation of an albumin-binding PET radiotracer 68Ga-NEB. J. Nucl. Med. 56, 1609-1614 (2015).

37. Harrell, M. I., Iritani, B. M. \& Ruddell, A. Lymph node mapping in the mouse. J. Immunol. Methods 332, 170-174 (2007).

38. Tsopelas, C. \& Sutton, R. Why certain dyes are useful for localizing the sentinel lymph node. J. Nucl. Med. 43, 1377-1382 (2002).

39. Zhang, W. et al. Potential applications of using $68 \mathrm{Ga}-$ Evans blue PET/CT in the evaluation of lymphatic disorder: preliminary observations. Clin. Nucl. Med. 41, 302 (2016).

40. Malaowalla, A. M. \& Fong, C. Toxicity of Evans blue dye in the monkey and tracing of it in the tooth pulp. Oral. Surg. Oral. Med. Oral. Pathol. 15, 1259-1263 (1962)

41. York, A. G. et al. Instant super-resolution imaging in live cells and embryos via analog image processing. Nat. Methods 10, 1122-1126 (2013).

42. Putta, M. R. et al. Peptide conjugation at the $5^{\prime}$-end of oligodeoxynucleotides abrogates toll-like receptor 9-mediated immune stimulatory activity. Bioconjug. Chem. 21, 39-45 (2010).

43. Liu, Y. et al. Stable Evans blue derived exendin-4 peptide for type 2 diabetes treatment. Bioconjug. Chem. 27, 54-58 (2016).

44. Chen, H. et al. Novel molecular "add-on" based on Evans Blue confers superior pharmacokinetics and transforms drugs to theranostic agents. J. Nucl. Med. 58, 590-597 (2016)

45. Heikenwalder, M. et al. Lymphoid follicle destruction and immunosuppression after repeated CpG oligodeoxynucleotide administration. Nat. Med. 10, 187-192 (2004).

46. Yang, B. et al. Single-cell phenotyping within transparent intact tissue through whole-body clearing. Cell 158, 945-958 (2014).

47. Leifer, C. A. et al. TLR9 is localized in the endoplasmic reticulum prior to stimulation. J. Immunol. 173, 1179-1183 (2004).

48. Saini, S. K. et al. Dipeptides promote folding and peptide binding of MHC class I molecules. Proc. Natl Acad. Sci. USA 110, 15383-15388 (2013).

49. Gros, A. et al. Prospective identification of neoantigen-specific lymphocytes in the peripheral blood of melanoma patients. Nat. Med. 22, 433-438 (2016).

50. Tanei, T., Leonard, F., Liu, X., Alexander, J. F. \& Saito, Y. Redirecting transport of nanoparticle albumin-bound paclitaxel to macrophages enhances therapeutic efficacy against liver metastases. Cancer Res. 76, 2 (2016).

51. Cullis, J. E. et al. Macropinocytosis of nab-paclitaxel drives macrophage activation in pancreatic cancer. Cancer Immunol. Res. 5, 182-190 (2017)

52. Liu, H., Kwong, B. \& Irvine, D. J. Membrane anchored immunostimulatory oligonucleotides for in vivo cell modification and localized immunotherapy. Angew. Chem. Int. Ed. Engl. 50, 7052-7055 (2011).

53. Lincoff, M. A. et al. Effect of the REG1 anticoagulation system versus bivalirudin on outcomes after percutaneous coronary intervention (REGULATE-PCI): a randomised clinical trial. Lancet 387, 349-356 (2016).

54. Sharma, P. \& Allison, J. P. The future of immune checkpoint therapy. Science 348, 56-61 (2015).

55. Carreno, B. M. et al. A dendritic cell vaccine increases the breadth and diversity of melanoma neoantigen-specific T cells. Science 348, 803-808 (2015).

56. Gubin, M. M. et al. Checkpoint blockade cancer immunotherapy targets tumour-specific mutant antigens. Nature 515, 577-581 (2014).

57. Tran, E., Robbins, P. F. \& Rosenberg, S. A. 'Final common pathway' of human cancer immunotherapy: targeting random somatic mutations. Nat. Immunol. 18, 255-262 (2017).

58. Cohen, C. J. et al. Isolation of neoantigen-specific T cells from tumor and peripheral lymphocytes. J. Clin. Invest. 125, 3981-3991 (2015).

59. Alexandrov, L. B. et al. Signatures of mutational processes in human cancer. Nature 500, 415-421 (2013).
60. Guex, N. \& Peitsch, M. C. SWISS-MODEL and the Swiss-PdbViewer: an environment for comparative protein modeling. Electrophoresis 18, 2714-2723 (1997).

61. Guex, N., Peitsch, M. C. \& Schwede, T. Automated comparative protein structure modeling with SWISS-MODEL and Swiss-PdbViewer: a historical perspective. Electrophoresis 30, S162-S173 (2009)

62. Vanommeslaeghe, K. et al. CHARMM general force field: a force field for drug like molecules compatible with the CHARMM all-atom additive biological force fields. J. Comput. Chem. 31, 671-690 (2010).

63. Perilla, J. R. et al. Molecular dynamics simulations of large macromolecular complexes. Curr. Opin. Struct. Biol. 31, 64-74 (2015).

64. Laskowski, R. A., MacArthur, M. W., Moss, D. S. \& Thornton, J. M. PROCHECK- a program to check the stereochemical quality of protein structures. J. Appl. Crystallogr. 26, 283-291 (1993).

65. Morris, G. M. et al. AutoDock4 and AutoDockTools4: automated docking with selective receptor flexibility. J. Comput. Chem. 30, 2785-2791 (2009).

66. Chen, K., Aowad, A. F., Adelstein, S. J. \& Kassis, A. I. Molecular-dockingguided design, synthesis, and biologic evaluation of radioiodinated quinazolinone prodrugs. J. Med. Chem. 50, 663-673 (2007).

\section{Acknowledgements}

We thank Dr Robert Langer (MIT) and Dr Henry Eden (NIBIB) for valuable suggestions and insightful comments on our manuscript. We thank Dr Xin Xu (NIAID) for research discussion and reagent contribution; Dr Meghan O. Altman (NIAID) for reagent contribution; Ms Lynne Holtzclaw and Dr Vincent Schram (NICHD) for their valuable expertise in tissue clearing and fluorescence microscopy; Dr Courtney Akitake (Carl Zeiss Microscopy LLC) and Mr Geoffrey Daniels (Leica Microsystems Inc.) for help with fluorescence microscopy; Mr Sam Darko (VRC of NIAID) for data analysis of RNA sequencing; Drs Jennie Garcia-Olivares (NIMH), Francisco J. Palma-Cerda (NINDS), and Joseph R Francica (NIAID) for help with BLI; and NIH Tetramer Core Facility for providing tetramers. This work was supported by Intramural Research Programs of National Institutes of Health, and National Science Foundation of China to Dr Yi Liu (81771900, 81601531, and BK20160755).

\section{Author contributions}

G.Z.: designed and conducted most experiments, analyzed and interpreted data, wrote manuscript; G.M.L.: designed experiments, immune analysis, exome sequencing, and discussion; O.J.: radiolabeling and PET; K.C.: molecular docking; H.Z.: photophysics of MEB-DNA; Y.L., F.Z., Z.W., L.L. and I.W.: reagent contribution; Y.M.: ESI-MS analysis; R.T., Z.W., N.L., Q.N. and S.C.: animals studies of PET and therapy; X.F. and A.J.: AFM; H.V. and H.S.: instant SIM; D.M.K.: antibody measurement and discussion; G.N. and B. Y.: interpreted data and revised manuscript; R.A.S.: supervision and discussion; X.C.: supervised all studies, designed experiments, interpreted data, and wrote manuscript.

\section{Additional information}

Supplementary Information accompanies this paper at https://doi.org/10.1038/s41467 017-02191-y.

Competing interests: G.Z. and X.C. are listed as inventors of a patent describing AlbiVax. The remaining authors declare no competing financial interests.

Reprints and permission information is available online at http://npg.nature.com/ reprintsandpermissions/

Publisher's note: Springer Nature remains neutral with regard to jurisdictional claims in published maps and institutional affiliations.

Open Access This article is licensed under a Creative Commons Attribution 4.0 International License, which permits use, sharing, adaptation, distribution and reproduction in any medium or format, as long as you give appropriate credit to the original author(s) and the source, provide a link to the Creative Commons license, and indicate if changes were made. The images or other third party material in this article are included in the article's Creative Commons license, unless indicated otherwise in a credit line to the material. If material is not included in the article's Creative Commons license and your intended use is not permitted by statutory regulation or exceeds the permitted use, you will need to obtain permission directly from the copyright holder. To view a copy of this license, visit http://creativecommons.org/ licenses/by/4.0/

(c) The Author(s) 2017 